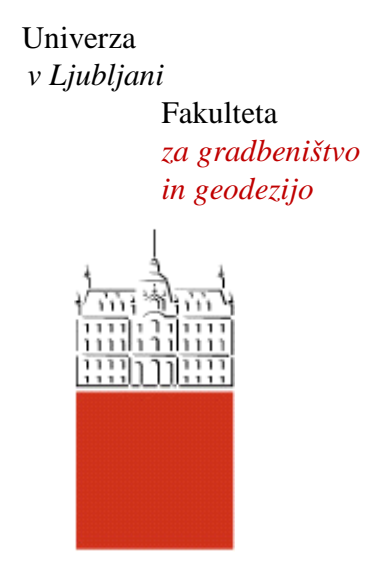

Jamova 2

1000 Ljubljana, Slovenija http://www3.fgg.uni-lj.si//

DRUGG - Digitalni repozitorij UL FGG http://drugg.fgg.uni-lj.si/

Ta članek je avtorjeva zadnja recenzirana različica, kot je bila sprejeta po opravljeni recenziji.

Prosimo, da se pri navajanju sklicujte na bibliografske podatke, kot je navedeno:
University
of Ljubljana

Faculty of

Civil and Geodetic

Engineering

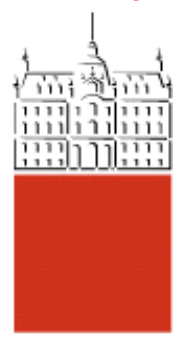

Jamova 2

SI - 1000 Ljubljana, Slovenia

http://www3.fgg.uni-lj.si/

DRUGG - The Digital Repository http://drugg.fgg.uni-lj.si/

This version of the article is author's manuscript as accepted for publishing after the review process.

When citing, please refer to the publisher's bibliographic information as follows:

Dujc, J., Brank, B., 2012. Stress resultant plasticity for shells revisited 247/248 : 146-165 http://www.sciencedirect.com/science/article/pii/S0045782512002320 


\title{
Stress resultant plasticity for shells revisited
}

\author{
Jaka Dujc, Boštjan Brank \\ University of Ljubljana, \\ Faculty of Civil and Geodetic Engineering, \\ Jamova 2, Ljubljana, Slovenia \\ e-mail: bbrank@ikpir.fgg.uni-lj.si
}

\begin{abstract}
In this work, we revisit the stress resultant elastoplastic geometrically exact shell finite element formulation that is based on the Ilyushin-Shapiro two-surface yield function with isotropic and kinematic hardening. The main focus is on implicit projection algorithms for computation of updated values of internal variables for stress resultant shell elastoplasticity. Four different algorithms are derived and compared. Three of them yield practically identical final results, yet they differ considerably in computational efficiency and implementation complexity, since they solve different sets of equations and they use different procedures that choose active yield surfaces. One algorithm does not provide acceptable accuracy. It turns out that the most simple and straightforward algorithm performs surprisingly well and efficiently. Several numerical examples are presented to illustrate the Ilyushin-Shapiro stress resultant shell formulation and the numerical performance of the presented integration algorithms.
\end{abstract}

Key words: shell, geometrically exact model, 4-node finite element, stress resultant plasticity, two-surface yield function, implicit integration algorithms

\section{Introduction}

The standard approach in the small strain elastoplastic analysis of shells is to define integration points in the through-the-thickness direction of the shell and to use stress-based elastoplastic constitutive equations; see e.g. [1], [4], [19], [21], [22]. The stress resultants are obtained by the through-the-thickness integration. Another approach in the small strain elastoplastic analysis of shells is based entirely on stress resultants, [10], [12], [15], [16], [17], [18], [20], [29], [34]. This is more in line with the geometrically exact shell theories involving inextensible director that treat the shell as a surface (with inextensible unit vector attached at each point) and extend the geometrical two-dimensionality into constitutive equations. Several geometrically exact shell formulations have been presented, e.g. [7], [23], [39], [40], [41]. Most of them use simple elastic stress resultant constitutive relations, since the derivation of inelastic (or nonlinear hyper-elastic) stress resultant constitutive relations for shells turns to be a non-trivial task. Even for the simplest case of the von Mises plasticity for solids, the counterpart shell stress resultant constitutive equations become rather complicated with two-surface yield function. This constitutive model is called Ilyushin if no hardening is included, and Ilyushin-Shapiro when hardening is taken into account [10]. The shell stress resultant counterparts of more complex solid inelastic constitutive models are very rare. We mention stress resultant models dealing with damage [17], anisotropic plasticity [20], and failure prediction of brittle shells [35]. In general, 
the inelastic (and also hyper-elastic) stress resultant constitutive models are very difficult (if not impossible in many cases) to derive from the solid constitutive models and also difficult to implement in the finite element framework to make them work in a good way.

With the stress resultant formulation, the information about the spread of plasticity through the shell thickness can be partially or completely lost. In the stress resultant space, a single point represents the state of the shell cross-section, which can be either elastic or plastic. Therefore, even for the pure (one dimensional) bending, when the shell cross-section is obviously partly elastic and partly plastic, the stress resultant plasticity will predict that the cross-section is either fully elastic or fully plastic. In contrast, the formulation with the stress-based elastoplasticity and through-the-thickness integration can capture the spread of plasticity through the thickness. In order to compensate this handicap of stress resultant formulations, Crisfield and Peng [12] introduced a parameter, associated with the equivalent plastic curvature, into the yield criterion. With this parameter, the effect of the spread of plasticity through the thickness can be somehow captured [6], [17], [20]. Another way to distinguish between the initiation of plastic deformations and the fully plastic state in stress resultant formulations was presented in [32] for plate bending problems by generalized plasticity with two hardening surfaces. We use the classical form of the Ilyushin-Shapiro plasticity that neglects the spread of plasticity through the thickness.

In many cases the difference between the classical Ilyushin-Shapiro stress resultant and the von Mises stress-based predictions is small. A shell structure resists to loads by exhibiting pure membrane, pure bending and combined membrane-bending states at its different regions [45]. For the pure membrane state, both formulations are identical. The difference for the pure bending state can be nicely illustrated by the plastic limit state analysis of plates in bending [6], [20]. The usual procedure in plastic limit state analysis is to neglect geometrically nonlinear effects and to assume perfectly plastic material without hardening. As shown in [6], the difference between the stress resultant and the stress-based formulations in pure bending state appears at the outset of plastification and soon afterwards. It becomes smaller with the increase of plastification across the structure that leads to very similar predictions of the limit load. Of course, the influence of chosen elastoplastic formulation on predicted shell response becomes harder to estimate when geometrically nonlinear effects (e.g. buckling) interact with plasticity. Our numerical simulations illustrate that also in such cases the predictions of stress resultant formulation compare reasonably good with the predictions of the stress-based formulation. We mention that the hardening in stress resultant plasticity is treated in a different way that in stress-based plasticity, which makes comparison of two formulations difficult.

In our opinion, the Ilyushin-Shapiro stress resultant plasticity is an attractive formulation for metal shells due to the fact that it is computationally cheaper and thus faster than the von Mises stress formulation with many integration points (at least five are usually used) in the throughthe-thickness direction. It can be effectively used, for example, for analysis of metal shells in civil engineering (silos, tanks, reservoirs, etc.). In many metal shell structures, its most parts are in the membrane state. Only small parts around concentrated and line forces, at supports, and at sharp changes of shell curvatures are in membrane-bending state with predominant bending. Thus, the inability of the stress resultant plasticity to take into account the spread of plasticity through the thickness should not be so important, since it influences only the bending dominating regions.

The above explains our motivation to revisit the Ilyushin-Shapiro stress resultant plasticity. In order to make it simple to implement, we studied several implicit return mapping algorithms. Our aim was to find the one that has good computational efficiency, is reliable and not too complex for the computer code implementation. Several integration algorithms have been already proposed: the shell version of the active set scheme for multi-surface plasticity [11] was derived in [10], the two-surface yield function was smoothed in [16] and [18] into a single one in order to 
use standard return mapping, a modified one-surface return mapping was used in [20]. In this work, we present and compare four different implicit projective algorithms that are combinations of two so-called options and two so-called procedures. First option condenses the complete set of nonlinear algebraic equations into a single nonlinear scalar equation for each yield surface, which can be done if the matrix that relates the stress resultants and the strains has constant entries, see e.g. [47], [28]. Second option solves the complete set of nonlinear algebraic equations related to the update of internal variables. We mention that the second option was elaborated in [28] for a general form of associated plasticity. The two derived procedures differ from one another by how the active set of yield surfaces is chosen. One of the procedures is similar to the active set two-yield surface scheme presented in [10]. We note that the most straightforward algorithm (i.e. combination of one option and one procedure) performs surprisingly well.

In the rest of this section, we will make some comments on thin shells, on shell finite elements and on geometrically exact shell model. The thin shell notion relates both to the character of the structural response and to the shell geometry itself. This is sometimes hidden, because it is very often checked by certain geometric ratio if a shell belongs to the class of thin shells. For example, [24, page 4] suggests that the shell is considered to be thin if its thickness is much smaller then its minimum radius of curvature at any point of its undeformed surface. It gives the approximate bonds of the ratio as 0.001 and 0.05 . Because the thin shell notion relates also to the structural behavior, these geometry-based bonds are of considerable latitude depending on loading, geometry, material, supports, etc. We emphasis that for thin shells the transverse shear part of their deformation energies is negligible with respect to the membrane and bending parts.

In this work, we use the geometrically exact, inextensible director shell theory with the Reissner-Mindlin kinematics (also called five-parameter theory [41]), which is valid for both thin and moderately thick shells. It assumes straight cross-sections, not necessarily perpendicular to the shell surface at deformed configuration, which is manifested in non-zero transverse shear strains that need to be related to the transverse shear forces through the constitutive relations. The elastoplastic constitutive relations are used for the transverse shear effects in this work. However, it would not be unreasonable to treat the transverse shear deformations as elastic when dealing with thin shells. Such an approach would have similarities with the quasi-Kirchhoff shell formulation [42], with the transverse shear modulus playing a role of the parameter that penalizes the Reissner-Mindlin kinematics to the Kirchhoff one. Another possibility, when considering thin shells, would be to remove the transverse shear strains from the formulation by changing the Reissner-Mindlin kinematics with the Kirchhoff one. Such a change comes along with the task of derivation of the corresponding working finite element of the discrete-Kirchhoff type, see [25] for shell element and [27] for plate element.

The shell finite element that we use is 4-node quadrilateral. As explained above, it is based on geometrically exact, inextensible director shell theory with Reissner-Mindlin kinematics. To avoid the transverse shear locking, the assumed natural strain (ANS) formulation [3] is applied, which is one of many different techniques for treatment the transverse shear locking in shell finite elements. Other representative techniques that have been developed over the years are the selective reduced integration, the enhanced assumed strain methods [30], the discrete gap methods [36], the linked-interpolation methods [27], the discrete-Kirchhoff formulations [25], to mention just a few. The 4-node quadrilateral also exhibits slight membrane locking, which can be cured by the enhanced assumed strain method [2], [26].

The notion geometrically exact shell model was introduced by Simo et al. [23], [10]. It describes a stress resultant shell theory that accurately describes large deformations and large rotations of the shell. The main characteristic of geometrically exact model is the presence of finite rotation parameters [5] in deformed configuration description. Over the years, the 
geometrically exact shell models have evolved into 3d-shell models [37], solid-shell models [2], [31], rotation-less shell models [43], and large rotation Kirchhoff models [25].

The rest of the paper is organized as follows. In section 2, the inelastic Ilyushin-Shapiro geometrically exact finite element shell formulation is briefly presented. In section 3, four integration algorithms for computation of updated values of internal variables are derived and discussed. Numerical examples are presented in section 4 , and conclusions are drawn in section 5 .

\section{Inelastic geometrically exact shell formulation}

In this section we present inelastic shell model that is formulated entirely in stress resultants and can accommodate large displacements and large rotations.

\subsection{Geometry, kinematics and strains}

We model a shell with its middle surface that has an inextensible unit vector (shell director) attached at its every point. The position vector of a material point of the initial stress-free shell configuration is then defined by

$$
\boldsymbol{X}\left(\xi^{1}, \xi^{2}, \xi\right)=\boldsymbol{\varphi}_{0}\left(\xi^{1}, \xi^{2}\right)+\xi \boldsymbol{T}\left(\xi^{1}, \xi^{2}\right), \quad\left(\xi^{1}, \xi^{2}\right) \in \mathcal{A}, \quad \xi \in \mathcal{F}
$$

Here, $\xi^{1}$ and $\xi^{2}$ are convective curvilinear coordinates that parametrize the midsurface; $\mathcal{A}$ is the domain of the parametrization; $\boldsymbol{T},\|\boldsymbol{T}\|=1$, is the shell director that coincides with the normal vector to the midsurface; and $\xi$ is through-the-thickness convective coordinate defined in the domain $\mathcal{F}=[-h / 2, h / 2]$, where $h$ represents initial shell thickness, here assumed to be constant. In what follows, we always determine the components of the above vectors with respect to the fixed orthonormal basis $\boldsymbol{e}_{i}=\boldsymbol{e}^{i}, i=1,2,3$, in the 3d space, i.e. $\boldsymbol{X}=X^{i} \boldsymbol{e}_{i}, \boldsymbol{\varphi}_{0}=\varphi_{0}^{i} \boldsymbol{e}_{i}, \boldsymbol{T}=T^{i} \boldsymbol{e}_{i}$. We further define the shell director as $\boldsymbol{T}=\boldsymbol{\Lambda}_{0} \boldsymbol{e}_{3}$, where $\boldsymbol{\Lambda}_{0}$ is a given (initial) rotation tensor, $\boldsymbol{\Lambda}_{0}^{-1}=\boldsymbol{\Lambda}_{0}^{T}, \operatorname{det} \boldsymbol{\Lambda}_{0}=1$. If one introduces at a point of midsurface an orthonormal basis $\widehat{\boldsymbol{e}}_{i}=\widehat{\boldsymbol{e}}^{i}$ as

$$
\widehat{\boldsymbol{e}}_{3} \equiv \boldsymbol{T}, \quad \widehat{\boldsymbol{e}}_{1} \perp \widehat{\boldsymbol{e}}_{3}, \quad\left\|\widehat{\boldsymbol{e}}_{1}\right\|=1, \quad \widehat{\boldsymbol{e}}_{2}=\widehat{\boldsymbol{e}}_{3} \times \widehat{\boldsymbol{e}}_{1}
$$

the rotation tensor $\boldsymbol{\Lambda}_{0}$ at that point can be represented as $\boldsymbol{\Lambda}_{0}=\left[\widehat{\boldsymbol{e}}_{1}, \widehat{\boldsymbol{e}}_{2}, \boldsymbol{T}\right]$. It is further assumed that the position vector to the material point in the deformed configuration is given by

$$
\boldsymbol{x}\left(\xi^{1}, \xi^{2}, \xi\right)=\underbrace{\left[\boldsymbol{\varphi}_{0}\left(\xi^{1}, \xi^{2}\right)+\boldsymbol{u}\left(\xi^{1}, \xi^{2}\right)\right]}_{\varphi\left(\xi^{1}, \xi^{2}\right)}+\xi \boldsymbol{t}\left(\xi^{1}, \xi^{2}\right), \quad\|\boldsymbol{t}\|=1
$$

In (3), $\boldsymbol{u}$ is a displacement vector of a midsurface point, and $\boldsymbol{t}$ is new position of shell director at the deformed configuration. We will define $t$ as the following sequence of two rotations $\boldsymbol{t}=\boldsymbol{\Lambda}_{0} \boldsymbol{\Lambda} \boldsymbol{e}_{3}$. The components of newly defined vectors in (3) are also determined with respect to the fixed orthonormal basis $\boldsymbol{e}_{i}$, i.e. $\boldsymbol{x}=x^{i} \boldsymbol{e}_{i}, \boldsymbol{\varphi}=\varphi^{i} \boldsymbol{e}_{i}, \boldsymbol{u}=u^{i} \boldsymbol{e}_{i}$, and $\boldsymbol{t}=t^{i} \boldsymbol{e}_{i}$. The rotation tensor $\boldsymbol{\Lambda}$ is viewed in this work as a function of a constrained rotation vector $\boldsymbol{\vartheta}$, i.e. $\boldsymbol{\Lambda}=\widetilde{\boldsymbol{\Lambda}}(\boldsymbol{\vartheta})$, see e.g. [5], [4], [7] for details. Since the rotation around the shell director (i.e. drilling rotation) plays no role in the present theory, the constrained rotation vector has only two components with respect to the basis $\widehat{\boldsymbol{e}}_{i}$, i.e. $\boldsymbol{\vartheta}=\vartheta^{\alpha} \widehat{\boldsymbol{e}}_{\alpha}, \alpha=1,2$. By using the Rodrigues formula for the representation of $\widetilde{\boldsymbol{\Lambda}}(\boldsymbol{\vartheta})$, one ends up with the following expression for $\boldsymbol{t}=\boldsymbol{t}(\boldsymbol{\vartheta})$, see [5]

$$
\boldsymbol{t}=\boldsymbol{\Lambda}_{0}\left(\cos \vartheta \boldsymbol{e}_{3}+\frac{\sin \vartheta}{\vartheta} \vartheta \times \boldsymbol{e}_{3}\right), \quad \vartheta=\|\vartheta\|
$$


The vectors of the convected basis $\boldsymbol{G}_{i}$ at the initial configuration are related to the position vector $\boldsymbol{X}$ and to the convected coordinates $\xi^{\alpha}, \xi$ as

$$
\boldsymbol{G}_{\alpha}=\frac{\partial \boldsymbol{X}}{\partial \xi^{\alpha}}=\frac{\partial \boldsymbol{\varphi}_{0}}{\partial \xi^{\alpha}}+\xi \frac{\partial \boldsymbol{T}}{\partial \xi^{\alpha}}, \quad \boldsymbol{G}_{3}=\frac{\partial \boldsymbol{X}}{\partial \xi}=\boldsymbol{T}
$$

Similarly, the vectors of the convected basis $\boldsymbol{g}_{i}$ at the deformed configuration are

$$
\boldsymbol{g}_{\alpha}=\frac{\partial \boldsymbol{x}}{\partial \xi^{\alpha}}=\frac{\partial \boldsymbol{\varphi}}{\partial \xi^{\alpha}}+\xi \frac{\partial \boldsymbol{t}}{\partial \xi^{\alpha}}, \quad \boldsymbol{g}_{3}=\frac{\partial \boldsymbol{x}}{\partial \xi}=\boldsymbol{t}
$$

The corresponding dual base vectors $\boldsymbol{G}^{i}$ and $\boldsymbol{g}^{i}$ are defined through the relationships $\boldsymbol{G}_{i} \cdot \boldsymbol{G}^{j}=\delta_{i}^{j}$ and $\boldsymbol{g}_{i} \cdot \boldsymbol{g}^{j}=\delta_{i}^{j}$, where $\delta_{i}^{j}$ is a Kronecker symbol. Note that $\boldsymbol{G}^{3}=\boldsymbol{G}_{3}$. The identity tensor of the shell reference configuration (or the shell metric tensor) is $\boldsymbol{G}=\boldsymbol{G}_{i} \otimes \boldsymbol{G}^{j}=G_{i j} \boldsymbol{G}^{i} \otimes \boldsymbol{G}^{j}$, where $G_{i j}=\boldsymbol{G}_{i} \cdot \boldsymbol{G}_{j}$. The differential volume element is given as $d V=\sqrt{G} d \xi d \xi^{1} d \xi^{2}$, where $\sqrt{G}=\boldsymbol{G}_{3} \cdot\left(\boldsymbol{G}_{1} \times \boldsymbol{G}_{2}\right)$. The base vectors at the reference midsurface and at the deformed midsurface are obtained by setting $\xi=0$ in (5) and (6), respectively, i.e. $\boldsymbol{A}_{i}=\left.\boldsymbol{G}_{i}\right|_{\xi=0}$, and $\boldsymbol{a}_{i}=\left.\boldsymbol{g}_{i}\right|_{\xi=0}$. The corresponding dual base vectors of $\boldsymbol{A}^{i}$ and $\boldsymbol{a}^{i}$ are defined as $\boldsymbol{A}_{i} \cdot \boldsymbol{A}^{j}=\delta_{i}^{j}$ and $\boldsymbol{a}_{i} \cdot \boldsymbol{a}^{j}=\delta_{i}^{j}$, respectively. Note that $\boldsymbol{A}^{3}=\boldsymbol{A}_{3}$. The identity (or metric) tensor of the shell reference midsurface is $\boldsymbol{A}=\boldsymbol{A}_{\alpha} \otimes \boldsymbol{A}^{\beta}=A_{\alpha \beta} \boldsymbol{A}^{\alpha} \otimes \boldsymbol{A}^{\beta}=A_{\alpha \beta}\left(\boldsymbol{A}^{\alpha}\right)_{\gamma}\left(\boldsymbol{A}^{\beta}\right)_{\delta} \boldsymbol{e}_{\gamma} \otimes \boldsymbol{e}_{\delta}=$ $\delta_{\alpha}^{\beta} \boldsymbol{e}_{\alpha} \otimes \boldsymbol{e}_{\beta}$, where $A_{\alpha \beta}=\boldsymbol{A}_{\alpha} \cdot \boldsymbol{A}_{\beta}$ and $A^{\alpha \beta}=\boldsymbol{A}^{\alpha} \cdot \boldsymbol{A}^{\beta}$. The differential surface element is given as $d A=\sqrt{A} d \xi^{1} d \xi^{2}$, where $\sqrt{A}=\left\|\boldsymbol{A}_{1} \times \boldsymbol{A}_{2}\right\|$. We can further define a tensor, called shifter, which transforms the base vectors of the midsurface to the base vectors of the shell body. The shifter of the shell reference configuration, denoted as $\boldsymbol{Z}$, shifts $\boldsymbol{A}_{i}$ and $\boldsymbol{A}^{i}$ to $\boldsymbol{G}_{i}$ and $\boldsymbol{G}^{i}$, respectively, i.e. $\boldsymbol{G}_{i}=\boldsymbol{Z} \boldsymbol{A}_{i}$ and $\boldsymbol{G}^{i}=\boldsymbol{Z}^{-T} \boldsymbol{A}^{i}$. In what follows, we assume that a shell is sufficiently thin that $\boldsymbol{Z} \approx \boldsymbol{I}$.

Having defined the base vectors, we can proceed with the expression for the deformation gradient

$$
\boldsymbol{F}=\frac{\partial \boldsymbol{x}}{\partial \boldsymbol{X}}=\frac{\partial \boldsymbol{x}}{\partial \xi^{i}}\left[\frac{\partial \boldsymbol{X}}{\partial \xi^{i}}\right]^{-1}=\boldsymbol{g}_{i} \otimes \boldsymbol{G}^{i}
$$

In (7) we used notation $\xi^{3}=\xi$. By knowing $\boldsymbol{F}$, we can obtain the components of the GreenLagrange strain tensor with respect to the convected basis $\boldsymbol{A}^{i}$

$$
\begin{aligned}
\boldsymbol{E} & =\frac{1}{2}\left(\boldsymbol{F}^{T} \boldsymbol{F}-\boldsymbol{G}\right)=\frac{1}{2}\left[\left(\boldsymbol{G}^{i} \otimes \boldsymbol{g}_{i}\right)\left(\boldsymbol{g}_{j} \otimes \boldsymbol{G}^{j}\right)-\boldsymbol{G}_{i} \cdot \boldsymbol{G}_{j}\left(\boldsymbol{G}^{i} \otimes \boldsymbol{G}^{j}\right)\right] \\
& =\frac{1}{2}\left(\boldsymbol{g}_{i} \cdot \boldsymbol{g}_{j}-\boldsymbol{G}_{i} \cdot \boldsymbol{G}_{j}\right) \boldsymbol{G}^{i} \otimes \boldsymbol{G}^{j}=E_{i j} \boldsymbol{G}^{i} \otimes \boldsymbol{G}^{j} \underbrace{\approx}_{\boldsymbol{Z}=\boldsymbol{I}} E_{i j} \boldsymbol{A}^{i} \otimes \boldsymbol{A}^{j}
\end{aligned}
$$

By evaluation of the dot products in (8) one can get the strains $E_{i j}$, which are varying quadratically with respect to the $\xi$ coordinate

$$
E_{i j}=\varepsilon_{i j}+\xi \kappa_{i j}+(\xi)^{2} \eta_{i j}
$$

Note, that $\varepsilon_{33}=\kappa_{33}=\eta_{\alpha 3}=\eta_{3 \alpha}=\eta_{33}=0$. In this work we will truncate the strains $E_{\alpha \beta}$ after the linear term, and the transverse shear strains $E_{\alpha 3}=E_{3 \alpha}$ after the constant term, i.e.

$$
E_{\alpha \beta} \rightarrow \varepsilon_{\alpha \beta}+\xi \kappa_{\alpha \beta}, \quad E_{\alpha 3} \rightarrow \varepsilon_{\alpha 3}
$$

The Green-Lagrange tensor that we will work with will have the following components in basis $\boldsymbol{A}^{i}$

$$
\boldsymbol{E} \approx E_{i j} \boldsymbol{A}^{i} \otimes \boldsymbol{A}^{j}=\varepsilon_{\alpha \beta} \boldsymbol{A}^{\alpha} \otimes \boldsymbol{A}^{\beta}+\xi \kappa_{\alpha \beta} \boldsymbol{A}^{\alpha} \otimes \boldsymbol{A}^{\beta}+\varepsilon_{\alpha 3}\left(\boldsymbol{A}^{\alpha} \otimes \boldsymbol{T}+\boldsymbol{T} \otimes \boldsymbol{A}^{\alpha}\right)
$$


Expressions for $\varepsilon_{\alpha \beta}, \kappa_{\alpha \beta}$ and $2 \varepsilon_{\alpha 3}$ in (10) are the classical expressions for the shell membrane, the shell bending and the shell transverse shear strains, respectively. Their explicit forms follow from using $\boldsymbol{g}_{i}$ and $\boldsymbol{G}_{i}$ in (8)

$$
\begin{aligned}
& \varepsilon_{\alpha \beta}=\frac{1}{2}\left(\boldsymbol{\varphi}_{, \alpha} \cdot \boldsymbol{\varphi}_{, \beta}-\boldsymbol{\varphi}_{0, \alpha} \cdot \boldsymbol{\varphi}_{0, \beta}\right), \quad 2 \varepsilon_{\alpha 3}=\widetilde{\gamma}_{\alpha}=\boldsymbol{\varphi}_{, \alpha} \cdot \boldsymbol{t}-\underbrace{\boldsymbol{\varphi}_{0, \alpha} \cdot \boldsymbol{T}}_{0} \\
& \kappa_{\alpha \beta}=\frac{1}{2}\left(\boldsymbol{\varphi}_{, \alpha} \cdot \boldsymbol{t}_{, \beta}+\boldsymbol{\varphi}_{, \beta} \cdot \boldsymbol{t}_{, \alpha}-\boldsymbol{\varphi}_{0, \alpha} \cdot \boldsymbol{T}_{, \beta}-\boldsymbol{\varphi}_{0, \beta} \cdot \boldsymbol{T}_{, \alpha}\right)
\end{aligned}
$$

For further use we will also express the components of $\boldsymbol{E}$ with respect to the orthonormal midsurface basis $\widehat{\boldsymbol{e}}_{i}$, defined above in (2)

$$
\boldsymbol{E} \approx \widehat{E}_{i j} \boldsymbol{A}^{i} \otimes \boldsymbol{A}^{j}=\widehat{\varepsilon}_{\alpha \beta} \widehat{\boldsymbol{e}}_{\alpha} \otimes \widehat{\boldsymbol{e}}_{\beta}+\xi \widehat{\kappa}_{\alpha \beta} \widehat{\boldsymbol{e}}_{\alpha} \otimes \widehat{\boldsymbol{e}}_{\beta}+\widehat{\varepsilon}_{\alpha 3}\left(\widehat{\boldsymbol{e}}_{\alpha} \otimes \boldsymbol{T}+\boldsymbol{T} \otimes \widehat{\boldsymbol{e}}_{\alpha}\right)
$$

The transformations between the strains (11) and the strains (13) take the following rules

$$
\widehat{\varepsilon}_{\gamma \delta}=\varepsilon_{\alpha \beta}\left(\widehat{\boldsymbol{e}}_{\gamma} \cdot \boldsymbol{A}^{\alpha}\right)\left(\widehat{\boldsymbol{e}}_{\delta} \cdot \boldsymbol{A}^{\beta}\right), \quad \widehat{\kappa}_{\gamma \delta}=\kappa_{\alpha \beta}\left(\widehat{\boldsymbol{e}}_{\gamma} \cdot \boldsymbol{A}^{\alpha}\right)\left(\widehat{\boldsymbol{e}}_{\delta} \cdot \boldsymbol{A}^{\beta}\right), \quad \widehat{\gamma}_{\gamma}=\widetilde{\gamma}_{\alpha}\left(\widehat{\boldsymbol{e}}_{\gamma} \cdot \boldsymbol{A}^{\alpha}\right)
$$

The stress resultants, which are energy-conjugated to the Green-Lagrange strains $\varepsilon_{\alpha \beta}, \kappa_{\alpha \beta}$ and $\widetilde{\gamma}_{\alpha}$, are the second Piola-Kirchhoff stress resultants $n^{\alpha \beta}, q^{\alpha}$ and $m^{\alpha \beta}$, respectively. The stress resultants $n^{\alpha \beta}, m^{\alpha \beta}$ and $q^{\alpha}$ are the classical (effective) shell membrane forces, the (symmetric) shell bending moments and the shell transverse shear forces. They are the components of the following second Piola-Kirchhoff stress resultant tensors resolved with respect to the $\boldsymbol{A}_{i}$ basis: the membrane force tensor $\boldsymbol{N}$, the transverse shear force tensor $\boldsymbol{Q}$ and the bending moment tensor $\boldsymbol{M}$

$$
\boldsymbol{N}=n^{\alpha \beta} \boldsymbol{A}_{\alpha} \otimes \boldsymbol{A}_{\beta}, \quad \boldsymbol{Q}=q^{\alpha}\left(\boldsymbol{A}_{\alpha} \otimes \boldsymbol{T}+\boldsymbol{T} \otimes \boldsymbol{A}_{\alpha}\right), \quad \boldsymbol{M}=m^{\alpha \beta} \boldsymbol{A}_{\alpha} \otimes \boldsymbol{A}_{\beta}
$$

Those stress resultant tensors can be also expressed with respect to the midsurface orthonormal basis $\widehat{\boldsymbol{e}}_{i}$ as

$$
\boldsymbol{N}=\widehat{n}^{\alpha \beta} \widehat{\boldsymbol{e}}_{\alpha} \otimes \widehat{\boldsymbol{e}}_{\beta}, \quad \boldsymbol{Q}=\widehat{q}^{\alpha}\left(\widehat{\boldsymbol{e}}_{\alpha} \otimes \boldsymbol{T}+\boldsymbol{T} \otimes \widehat{\boldsymbol{e}}_{\alpha}\right), \quad \boldsymbol{M}=\widehat{m}^{\alpha \beta} \widehat{\boldsymbol{e}}_{\alpha} \otimes \widehat{\boldsymbol{e}}_{\beta}
$$

The transformation of the stress resultants, defined with respect to $\boldsymbol{A}_{i}$ basis, to stress resultants, defined with respect to the midsurface orthonormal basis $\widehat{\boldsymbol{e}}_{i}$, is given as

$$
\widehat{n}^{\gamma \delta}=n^{\alpha \beta}\left(\widehat{\boldsymbol{e}}_{\gamma} \cdot \boldsymbol{A}_{\alpha}\right)\left(\widehat{\boldsymbol{e}}_{\delta} \cdot \boldsymbol{A}_{\beta}\right), \quad \widehat{m}^{\gamma \delta}=m^{\alpha \beta}\left(\widehat{\boldsymbol{e}}_{\gamma} \cdot \boldsymbol{A}_{\alpha}\right)\left(\widehat{\boldsymbol{e}}_{\delta} \cdot \boldsymbol{A}_{\beta}\right), \quad \widehat{q}^{\gamma}=q^{\alpha}\left(\widehat{\boldsymbol{e}}_{\gamma} \cdot \boldsymbol{A}_{\alpha}\right)
$$

\subsection{Variational formulation}

To simplify the notation, we will use in what follows the strains and the stress resultants resolved with respect to the local orthonormal basis $\widehat{e}_{i}$. Those strains will be collected into the following vectors

$$
\boldsymbol{\varepsilon}=\left[\boldsymbol{\epsilon}^{T}, \boldsymbol{\kappa}^{T}, \widetilde{\boldsymbol{\gamma}}^{T}\right]^{T}, \quad \boldsymbol{\epsilon}=\left[\widehat{\varepsilon}_{11}, \widehat{\varepsilon}_{22}, 2 \widehat{\varepsilon}_{12}\right]^{T}, \quad \boldsymbol{\kappa}=\left[\widehat{\kappa}_{11}, \widehat{\kappa}_{22}, 2 \widehat{\kappa}_{12}\right]^{T}, \quad \widetilde{\gamma}=\left[\widehat{\gamma}_{1}, \widehat{\gamma}_{2}\right]^{T}
$$

and the stress resultants will be collected as

$$
\boldsymbol{\sigma}=\left[\boldsymbol{n}^{T}, \boldsymbol{m}^{T}, \boldsymbol{q}^{T}\right]^{T}, \quad \boldsymbol{n}=\left[\widehat{n}_{11}, \widehat{n}_{22}, \widehat{n}_{12}\right]^{T}, \quad \boldsymbol{m}=\left[\widehat{m}_{11}, \widehat{m}_{22}, \widehat{m}_{12}\right]^{T}, \quad \boldsymbol{q}=\left[\widehat{q}_{1}, \widehat{q}_{2}\right]^{T}
$$

We will further define the virtual work equation, which will represent the starting point for the finite element discretization. Before defining the virtual work equation, we need to express the 
virtual strains. Those are the strains that arise due to variation of displacement vector $\boldsymbol{u}$ for virtual displacement vector $\delta \boldsymbol{u}$, and due to variation of rotation vector $\boldsymbol{\vartheta}$ for virtual rotation vector $\delta \boldsymbol{\vartheta}$. By introducing $\boldsymbol{u} \rightarrow \boldsymbol{u}+\omega \delta \boldsymbol{u}$ and $\boldsymbol{\vartheta} \rightarrow \boldsymbol{\vartheta}+\boldsymbol{\omega} \delta \boldsymbol{\vartheta}$ into the expressions for strains in (18), one can derive vector of virtual strains as

$$
\delta \varepsilon=\left.\frac{d}{d \omega} \boldsymbol{\varepsilon}(\boldsymbol{u}+\omega \delta \boldsymbol{u}, \boldsymbol{\vartheta}+\omega \delta \boldsymbol{\vartheta})\right|_{\omega=0}=\left[\delta \boldsymbol{\epsilon}^{T}, \delta \boldsymbol{\kappa}^{T}, \delta \widetilde{\gamma}^{T}\right]^{T}
$$

where

$$
\delta \boldsymbol{\epsilon}=\left[\delta \widehat{\varepsilon}_{11}, \delta \widehat{\varepsilon}_{22}, 2 \delta \widehat{\varepsilon}_{12}\right]^{T}, \quad \delta \boldsymbol{\kappa}=\left[\delta \widehat{\kappa}_{11}, \delta \widehat{\kappa}_{22}, 2 \delta \widehat{\kappa}_{12}\right]^{T}, \quad \delta \widetilde{\gamma}=\left[\delta \widehat{\gamma}_{1}, \delta \widehat{\gamma}_{2}\right]^{T}
$$

Explicit expressions for virtual strains in (21) are (see (14))

$$
\delta \widehat{\varepsilon}_{\gamma \delta}=\delta \varepsilon_{\alpha \beta}\left(\widehat{\boldsymbol{e}}_{\gamma} \cdot \boldsymbol{A}^{\alpha}\right)\left(\widehat{\boldsymbol{e}}_{\delta} \cdot \boldsymbol{A}^{\beta}\right), \quad \delta \widehat{\kappa}_{\gamma \delta}=\delta \kappa_{\alpha \beta}\left(\widehat{\boldsymbol{e}}_{\gamma} \cdot \boldsymbol{A}^{\alpha}\right)\left(\widehat{\boldsymbol{e}}_{\delta} \cdot \boldsymbol{A}^{\beta}\right), \quad \delta \widehat{\gamma}_{\gamma}=\delta \widetilde{\gamma}_{\alpha}\left(\widehat{\boldsymbol{e}}_{\gamma} \cdot \boldsymbol{A}^{\alpha}\right)
$$

where $($ see $(12))$

$$
\begin{aligned}
\delta \varepsilon_{\alpha \beta} & =\frac{1}{2}\left(\delta \boldsymbol{u}_{, \alpha} \cdot \boldsymbol{\varphi}_{, \beta}+\boldsymbol{\varphi}_{, \alpha} \cdot \delta \boldsymbol{u}_{, \beta}\right), \quad \delta \widetilde{\gamma}_{\alpha}=\delta \boldsymbol{u}_{, \alpha} \cdot \boldsymbol{t}+\boldsymbol{\varphi}_{, \alpha} \cdot \delta \boldsymbol{t} \\
\delta \kappa_{\alpha \beta} & =\frac{1}{2}\left(\delta \boldsymbol{u}_{, \alpha} \cdot \boldsymbol{t}_{, \beta}+\boldsymbol{\varphi}_{, \alpha} \cdot \delta \boldsymbol{t}_{, \beta}+\delta \boldsymbol{u}_{, \beta} \cdot \boldsymbol{t}_{, \alpha}+\boldsymbol{u}_{, \beta} \cdot \delta \boldsymbol{t}_{, \alpha}\right)=\delta \boldsymbol{u}_{, \alpha} \cdot \boldsymbol{t}_{, \beta}+\boldsymbol{\varphi}_{, \alpha} \cdot \delta \boldsymbol{t}_{, \beta}
\end{aligned}
$$

and $\delta \boldsymbol{t}=\left.\frac{d}{d \omega} \boldsymbol{t}(\boldsymbol{\vartheta}+\omega \delta \boldsymbol{\vartheta})\right|_{\omega=0}$.

The virtual work equation can now be written as the weak form of the shell problem

$$
G(\boldsymbol{u}, \boldsymbol{\vartheta} ; \delta \boldsymbol{u}, \delta \boldsymbol{\vartheta} ;(\circ))=\int_{A} \delta \boldsymbol{\varepsilon}^{T}(\boldsymbol{u}, \boldsymbol{\vartheta} ; \delta \boldsymbol{u}, \delta \boldsymbol{\vartheta}) \boldsymbol{\sigma}(\boldsymbol{u}, \boldsymbol{\vartheta},(\circ)) d A-G_{\text {ext }}(\delta \boldsymbol{u}, \delta \boldsymbol{\vartheta})=0
$$

where the expression under the integral sign represents the virtual work of internal forces and $G_{\text {ext }}$ represents the virtual work of external forces. An empty slot (o) represents internal variables of an inelastic constitutive model. The stress resultants $\boldsymbol{\sigma}$ are functions of those variables. Since we consider here a shell model with inelastic constitutive relations, we assume dependency of the displacements $\boldsymbol{u}$, the rotation vector $\boldsymbol{\vartheta}$, the strains $\boldsymbol{\varepsilon}$ and the stress resultants $\boldsymbol{\sigma}$ on pseudo-time parameter $t \in[0, T]$.

\subsection{Solution of the discretized weak form}

The solution of the weak form (24) should provide displacements of the shell midsurface and rotation of the shell director. It is obtained in the following manner. The weak form (24), is first discretized in space by using the finite element method. Then, the solution of the discretized weak form is searched for at discrete pseudo-time points $0<t_{1}<\ldots t_{n}<t_{n+1} \ldots<T$. The value of $(\diamond)$ at $t_{n+1}$ will be denoted as $(\diamond)_{n+1}$. The space discretization reveals that the solution of discretized form of (24) can be obtained by the operator split method, e.g. [14]. Namely, the solution search for displacements and rotation vector at discrete (nodal) points at $t_{n+1}$ can be performed separately (but not independently) from the solution of inelastic constitutive equations to get internal variables and stress resultants $\boldsymbol{\sigma}_{n+1}$ at the same pseudo-time instant.

Let us now shortly describe the solution search for displacements and rotation vector by assuming that the best guess for $\boldsymbol{\sigma}_{n+1}$ is known data. We use the superscript $(\odot)^{h}$ to denote that the function $(\odot)$ has been approximated in space by using finite element interpolation functions and unknown nodal parameters. After discretization the equation (24) turns to

$$
\begin{aligned}
& G^{h}\left(\boldsymbol{u}_{n+1}^{h}, \boldsymbol{t}^{h}\left(\boldsymbol{\vartheta}_{n+1}\right) ; \delta \boldsymbol{u}^{h}, \delta \boldsymbol{t}^{h}\left(\boldsymbol{\vartheta}_{n+1}, \delta \boldsymbol{\vartheta}\right)\right)= \\
& =\int_{A^{h}} \delta \boldsymbol{\varepsilon}^{h, T}\left(\boldsymbol{u}_{n+1}^{h}, \boldsymbol{\vartheta}_{n+1} ; \delta \boldsymbol{u}^{h}, \delta \boldsymbol{\vartheta}\right) \boldsymbol{\sigma}_{n+1}\left(\boldsymbol{u}_{n+1}^{h}, \boldsymbol{\vartheta}_{n+1},(\circ)_{n+1}\right) d A-G_{e x t}^{h}\left(\delta \boldsymbol{u}^{h}, \delta \boldsymbol{\vartheta}\right)=0
\end{aligned}
$$


Eq. (25) provides a set of highly nonlinear equations with $\boldsymbol{u}_{n+1}^{h}$ and $\boldsymbol{\vartheta}_{n+1}$ as the unknowns. Linearization of (25) around the current iterative state $\boldsymbol{u}_{n+1}^{h}, \boldsymbol{t}^{h}\left(\boldsymbol{\vartheta}_{n+1}\right)$ needs to be performed in order to get a solution by Newton-Raphson iterative method . One can derive linearized form of $G^{h}$ by introducing the following displacements $\boldsymbol{u}_{n+1}^{h} \rightarrow \boldsymbol{u}_{n+1}^{h}+\omega \Delta \boldsymbol{u}_{n+1}^{h}$ and the following rotation vector $\boldsymbol{\vartheta}_{n+1} \rightarrow \boldsymbol{\vartheta}_{n+1}+\omega \Delta \boldsymbol{\vartheta}_{n+1}$ into $G^{h}$ and by using

$$
\Delta G^{h}=\left.\frac{d}{d \omega} G^{h}\left(\boldsymbol{u}_{n+1}^{h}+\omega \Delta \boldsymbol{u}_{n+1}^{h}, \boldsymbol{\vartheta}_{n+1}+\omega \Delta \boldsymbol{\vartheta}_{n+1}\right)\right|_{\omega=0}
$$

It follows from (26) that

$$
\begin{aligned}
\Delta G^{h}= & \int_{A^{h}} \Delta \delta \varepsilon^{h, T}\left(\Delta \boldsymbol{u}_{n+1}^{h}, \Delta \boldsymbol{\vartheta}_{n+1} ; \delta \boldsymbol{u}^{h}, \delta \boldsymbol{\vartheta}\right) \boldsymbol{\sigma}_{n+1}\left(\boldsymbol{u}_{n+1}^{h}, \boldsymbol{\vartheta}_{n+1},(\circ)_{n+1}\right) d A+ \\
& \int_{A^{h}} \delta \boldsymbol{\varepsilon}^{h, T}\left(\boldsymbol{u}_{n+1}^{h}, \boldsymbol{\vartheta}_{n+1} ; \delta \boldsymbol{u}^{h}, \delta \boldsymbol{\vartheta}\right) \frac{d \boldsymbol{\sigma}_{n+1}}{d \boldsymbol{\varepsilon}_{n+1}^{h}} \Delta \boldsymbol{\varepsilon}_{n+1}^{h, T}\left(\boldsymbol{u}_{n+1}^{h}, \boldsymbol{\vartheta}_{n+1} ; \Delta \boldsymbol{u}_{n+1}^{h}, \Delta \boldsymbol{\vartheta}_{n+1}\right) d A
\end{aligned}
$$

where $\Delta \delta \varepsilon^{h}$ and $\Delta \varepsilon_{n+1}^{h}$ are defined in a similar way as $\Delta G^{h}$ in (26). Eq. (27) shows that the linearized discrete weak form is split into two parts; the first one is called geometric and the second one material. The $d \boldsymbol{\sigma}_{n+1} / d \varepsilon_{n+1}^{h}$ is called the consistent tangent modulus.

\subsection{Stress-resultant constitutive equations for elastoplasticity}

We will consider in this section the stress resultant elastoplasticity for shells. It will be based on Ilyushin-Shapiro multi-surface yield function, which is a stress resultant approximation of the classical von Mises yield function. The considered yield function takes into account both isotropic and kinematic hardening. The internal variables, describing the irreversible nature of the plastic process, are chosen as: the plastic strain $\varepsilon^{p}=\left[\boldsymbol{\epsilon}^{p, T}, \boldsymbol{\kappa}^{p, T}, \widetilde{\gamma}^{p, T}\right]^{T}$, the scalar parameter $\xi^{I}$, which controls the isotropic hardening mechanism, and the strain-like parameters $\varkappa=\left[\varkappa_{11}^{n}, \varkappa_{22}^{n}, \varkappa_{12}^{n}, \varkappa_{11}^{m}, \varkappa_{22}^{m}, \varkappa_{12}^{m}, \varkappa_{13}^{q}, \varkappa_{23}^{q}\right]^{T}$, which control the kinematic hardening mechanism. Those variables, strains $\varepsilon$ and stress resultants $\boldsymbol{\sigma}$ are functions of pseudo-time $t$, i.e. $\varepsilon^{p}=\varepsilon^{p}(t)$, $\xi^{I}=\xi^{I}(t), \varkappa=\varkappa(t), \varepsilon=\varepsilon(t)$ and $\boldsymbol{\sigma}=\boldsymbol{\sigma}(t)$.

A usual additive split of reversible (elastic) and irreversible (plastic) strains is assumed

$$
\varepsilon=\varepsilon^{e}+\varepsilon^{p}
$$

where $\boldsymbol{\varepsilon}^{e}=\left[\boldsymbol{\epsilon}^{e, T}, \boldsymbol{\kappa}^{e, T}, \widetilde{\boldsymbol{\gamma}}^{e, T}\right]^{T}$. The strain energy function is assumed to be of the following (quadratic) form

$$
\psi\left(\varepsilon^{e}, \xi^{I}, \varkappa\right)=\frac{1}{2} \varepsilon^{e, T} \boldsymbol{C} \varepsilon^{e}+\Xi\left(\xi^{I}\right)+\frac{1}{2} \varkappa^{T} \boldsymbol{D} \varkappa
$$

By assuming isotropic elastic response, $\boldsymbol{C}$ is given as

$$
\begin{aligned}
\boldsymbol{C} & =\operatorname{diag}\left[\boldsymbol{C}^{n}, \boldsymbol{C}^{b}, \boldsymbol{C}^{s}\right], \quad \boldsymbol{C}^{n}=h \overline{\boldsymbol{C}}, \quad \boldsymbol{C}^{b}=\frac{h^{3}}{12} \overline{\boldsymbol{C}} \\
\overline{\boldsymbol{C}} & =\frac{E}{\left(1-\nu^{2}\right)}\left[\begin{array}{ccc}
1 & \nu & 0 \\
\nu & 1 & 0 \\
0 & 0 & \frac{1-\nu}{2}
\end{array}\right], \quad \boldsymbol{C}^{s}=k^{s}\left[\begin{array}{cc}
1 & 0 \\
0 & 1
\end{array}\right]
\end{aligned}
$$

The constants in (30) are: $E$ is elastic modulus, $\nu$ is Poisson's ratio, $h$ is shell thickness, $k^{s}=c G h$, $G=\frac{E}{2(1+\nu)}$ is shear modulus and $c$ is shear correction factor, usually set to $5 / 6$ for an isotropic material. Matrix $\boldsymbol{D}$ in $(29)$ is $\boldsymbol{D}=\frac{2}{3} H_{k i n} \boldsymbol{I}_{8}$, where $\boldsymbol{I}_{8}=\operatorname{diag}[1,1,1,1,1,1,1,1]$. In (29) we have assumed a general (nonlinear) form of isotropic hardening and a linear form of kinematic 
hardening with hardening modulus $H_{k i n}$. We denote the stress-like internal variables, which correspond to the strain-like internal variables $\xi^{I}$ and $\varkappa$, as $q$ and $\boldsymbol{\alpha}$, respectively. These dual variables are used to define Ilyushin-Shapiro two-surface yield function $\phi=\phi\left(\phi_{1}, \phi_{2}\right)=0$. A sketch of a two-surface yield function is presented in Figure 1. The elastic domain is defined with $\phi_{1}<0 \cap \phi_{2}<0$ where $\phi<0$, while the plastic domain is defined with $\left(\phi_{1}<0 \cap \phi_{2}=0\right) \cup\left(\phi_{1}=\right.$ $\left.0 \cap \phi_{2}<0\right) \cup\left(\phi_{1}=0 \cap \phi_{2}=0\right)$ where $\phi=0$. The non-dimensional forms ob $\phi_{1}$ and $\phi_{2}$ can be

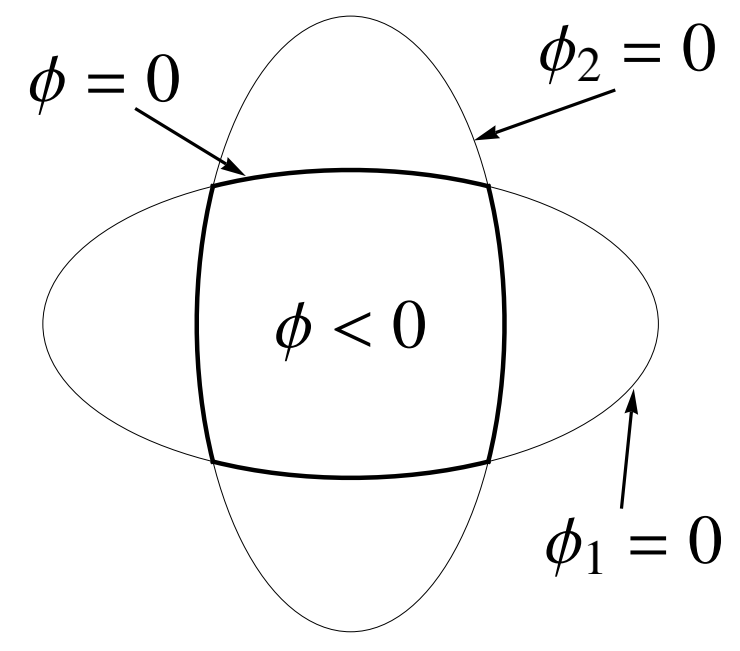

Figure 1: A sketch of a two-surface yield function

written as

$$
\phi_{\mu}(\boldsymbol{\sigma}, q, \boldsymbol{\alpha})=(\boldsymbol{\sigma}+\boldsymbol{\alpha})^{T} \boldsymbol{A}_{\mu}(\boldsymbol{\sigma}+\boldsymbol{\alpha})-\left(1-\frac{q}{\sigma_{y}}\right)^{2} \quad \mu=1,2
$$

Here, $\boldsymbol{\sigma}$ is defined in (19), $\boldsymbol{\alpha}$ (the negative of the back stress resultants) is defined as $\boldsymbol{\alpha}=$ $\left[\boldsymbol{\alpha}^{n, T}, \boldsymbol{\alpha}^{m, T}, \boldsymbol{\alpha}^{q, T}\right]^{T}$, where $\boldsymbol{\alpha}^{n}=\left[\alpha_{11}^{n}, \alpha_{22}^{n}, \alpha_{12}^{n}\right]^{T}, \boldsymbol{\alpha}^{m}=\left[\alpha_{11}^{m}, \alpha_{22}^{m}, \alpha_{12}^{m}\right]^{T}, \boldsymbol{\alpha}^{q}=\left[\alpha_{13}^{q}, \alpha_{23}^{q}\right]^{T}$ and $\sigma_{y}$ is uniaxial yield stress. The matrix $\boldsymbol{A}_{\mu}$ for isotropic plastic response equals to

$$
\begin{aligned}
& \boldsymbol{A}_{\mu}=\left[\begin{array}{lll}
\frac{1}{n_{0}^{2}} \boldsymbol{P} & \frac{\operatorname{sign}(\mu)}{2 \sqrt{3} n_{0} m_{0}} \boldsymbol{P} & \mathbf{0} \\
\frac{\operatorname{sign}(\mu)}{2 \sqrt{3} n_{0} m_{0}} \boldsymbol{P} & \frac{1}{m_{0}^{2}} \boldsymbol{P} & \mathbf{0} \\
\mathbf{0} & \mathbf{0} & \frac{1}{q_{0}^{2}} \boldsymbol{I}_{2}
\end{array}\right], \\
& \boldsymbol{P}=\frac{1}{2}\left[\begin{array}{ccc}
2 & -1 & 0 \\
-1 & 2 & 0 \\
0 & 0 & 6
\end{array}\right], \quad \operatorname{sign}(\mu)=\left\{\begin{array}{l}
+1 \text { if } \mu=1 \\
-1 \text { if } \mu=2
\end{array}\right.
\end{aligned}
$$

Here, $n_{0}, m_{0}$ and $q_{0}$ are the yield parameters associated with membrane extension, bending and transverse shear, respectively. They are usually set to the fully plastic uniaxial membrane force $n_{0}=\sigma_{y} h$, fully plastic uniaxial bending moment $m_{0}=\frac{\sigma_{y} h^{2}}{4}$ and fully plastic uniaxial transverse shear force $q_{0}=\frac{\sigma_{y} h}{\sqrt{3}}$. For further discussion on stress resultant yield functions for shells see [12], [10] and [13].

Having defined internal variables, strain energy function and yield function, we proceed with derivation of the remaining ingredients of the stress resultant elastoplasticity for shells. For the 
isothermal case we can write the following rate of material dissipation

$$
\mathcal{D}=\boldsymbol{\sigma}^{T} \dot{\varepsilon}-\frac{d}{d t} \psi\left(\varepsilon^{e}, \xi^{I}, \varkappa\right)=\boldsymbol{\sigma}^{T} \dot{\varepsilon}-\left(\frac{\partial \psi}{\partial \varepsilon^{e}}\right)^{T}\left(\dot{\varepsilon}-\dot{\varepsilon}^{p}\right)-\frac{\partial \psi}{\partial \xi^{I}} \dot{\xi}^{I}-\left(\frac{\partial \psi}{\partial \varkappa}\right)^{T} \dot{\varkappa} \geqslant 0
$$

which is assumed to be non-negative. In $(33)(\dot{0})=\frac{\partial(\circ)}{\partial t}$. Note that equation (33) can be derived from the second law of thermodynamics, see e.g. [10]. By assuming that the elastic process is non-dissipative (i.e. the state variables do not change during that process and $\mathcal{D}=0$ ) one has

$$
\boldsymbol{\sigma}=\frac{\partial \psi}{\partial \varepsilon^{e}}=\boldsymbol{C} \varepsilon^{e}
$$

By further consideration of (33) one can define the dual variables, i.e. the hardening variable $q$ and the variables that control kinematic hardening $\boldsymbol{\alpha}$, as

$$
q=-\frac{\partial \psi}{\partial \xi^{I}}=-\frac{d \Xi\left(\xi^{I}\right)}{d \xi^{I}}=-\Xi^{\prime}\left(\xi^{I}\right), \quad \boldsymbol{\alpha}=-\frac{\partial \psi}{\partial \varkappa}=-\boldsymbol{D} \varkappa
$$

If we consider the quadratic form of $\Xi=\frac{1}{2} K_{h} \xi^{I^{2}}$ we obtain the case of linear isotropic hardening

$$
q=-K_{h} \xi^{I}
$$

where $K_{h}$ is the isotropic hardening modulus. By using (34) and (35) in (33) we obtain the reduced material dissipation (i.e. the dissipation of the plastic process) as

$$
\mathcal{D}^{p}=\boldsymbol{\sigma}^{T} \dot{\varepsilon}^{p}+q \dot{\xi^{I}}+\boldsymbol{\alpha}^{T} \dot{\boldsymbol{\varkappa}} \geqslant 0
$$

The principle of maximum plastic dissipation states that among all the dual variables $(\boldsymbol{\sigma}, q, \boldsymbol{\alpha})$ that satisfy the yield criteria, one should choose those that maximize plastic dissipation. The problem can be written in the following form: Find minimum of $\mathcal{L}^{p}\left(\boldsymbol{\sigma}, q, \boldsymbol{\alpha}, \dot{\gamma}_{\mu}\right)$, where

$$
\mathcal{L}^{p}\left(\boldsymbol{\sigma}, q, \boldsymbol{\alpha}, \dot{\gamma}_{1}, \dot{\gamma}_{2}\right)=-\mathcal{D}^{p}(\boldsymbol{\sigma}, q, \boldsymbol{\alpha})+\sum_{\mu=1}^{2} \dot{\gamma}_{\mu} \phi_{\mu}(\boldsymbol{\sigma}, q, \boldsymbol{\alpha})
$$

and $\dot{\gamma}_{\mu} \geqslant 0$ play the role of Lagrange multipliers. From the above minimization problem and (31) we obtain explicit forms of evolution equations for the internal variables

$$
\begin{aligned}
\frac{\partial \mathcal{L}^{p}}{\partial \boldsymbol{\sigma}} & =-\dot{\boldsymbol{\varepsilon}}^{p}+\sum_{\mu=1}^{2} \dot{\gamma}_{\mu} \frac{\partial \phi_{\mu}}{\partial \boldsymbol{\sigma}}=0 \Longrightarrow \dot{\boldsymbol{\varepsilon}}^{p}=\sum_{\mu=1}^{2} \dot{\gamma}_{\mu} \underbrace{2 \boldsymbol{A}_{\mu}(\boldsymbol{\sigma}+\boldsymbol{\alpha})}_{\boldsymbol{\nu}_{\mu}} \\
\frac{\partial \mathcal{L}^{p}}{\partial q} & =-\dot{\xi}^{I}+\sum_{\mu=1}^{2} \dot{\gamma}_{\mu} \frac{\partial \phi_{\mu}}{\partial q}=0 \Longrightarrow \dot{\xi}^{I}=\sum_{\mu=1}^{2} \dot{\gamma}_{\mu}^{\frac{2}{\sigma_{y}}\left(1-\frac{q}{\sigma_{y}}\right)} \\
\frac{\partial \mathcal{L}^{p}}{\partial \boldsymbol{\alpha}} & =-\dot{\boldsymbol{\varkappa}}+\sum_{\mu=1}^{2} \dot{\gamma}_{\mu} \frac{\partial \phi_{\mu}}{\partial \boldsymbol{\alpha}}=0 \Longrightarrow \dot{\boldsymbol{\varkappa}}=\sum_{\mu=1}^{2} \dot{\gamma}_{\mu} \underbrace{2 \boldsymbol{A}_{\mu}(\boldsymbol{\sigma}+\boldsymbol{\alpha})}_{\boldsymbol{\nu}_{\mu}}
\end{aligned}
$$

Note that $\dot{\varkappa}=\dot{\varepsilon}^{p}$. The loading/unloading (Kuhn-Tucker) conditions follow from the demands that $\dot{\gamma}_{\mu}$ are non-negative, that $\phi_{\mu}$ are non-positive, and that the plastic dissipation $\mathcal{D}^{p}$ equals zero for elastic process when $\phi_{\mu}<0$

$$
\dot{\gamma}_{\mu} \geqslant 0, \quad \phi_{\mu} \leqslant 0, \quad \dot{\gamma}_{\mu} \phi_{\mu}=0
$$

In addition to (40) we have the condition $\dot{\phi}_{\mu}=0$ if $\dot{\gamma}_{\mu}>0$ (the consistency condition). It guarantees the admissibility of the subsequent state in the case of change of state variables. 


\subsection{Space-domain discretization}

Let the initial shell midsurface $A$ be discretized by $n_{e l}$ non-overlapping elements with $n_{e n}$ nodes such that $A \approx \bigcup_{e=1}^{n_{e l}} A_{e}=A^{h}$. Over the element domain $A_{e}$ the initial shell configuration (the midsurface and the shell director) are interpolated as

$$
\boldsymbol{\varphi}_{0}^{h}\left(\xi^{1}, \xi^{2}\right)=\sum_{a=1}^{n_{e n}} N_{a}\left(\xi^{1}, \xi^{2}\right) \boldsymbol{\varphi}_{0 a}, \quad \boldsymbol{T}^{h}\left(\xi^{1}, \xi^{2}\right)=\sum_{a=1}^{n_{e n}} N_{a}\left(\xi^{1}, \xi^{2}\right) \boldsymbol{T}_{a}
$$

where $(\circ)_{a}$ are the corresponding nodal values. In this work we choose $n_{e n}=4$ and the bilinear shape functions $N_{a}\left(\xi^{1}, \xi^{2}\right)$, defined over the square domain $\mathcal{A}_{e}=[-1,1] \times[-1,1]$. Note, that $\boldsymbol{T}_{a}$ is chosen to coincide with the normal vector to a given shell midsurface at that nodal point. However, due to the bi-linear interpolation (41) $\boldsymbol{T}^{h}$ is only approximately perpendicular to the base vectors $\boldsymbol{A}_{\alpha}^{h}=\partial \varphi_{0}^{h} / \partial \xi^{\alpha}$. The interpolation of the shell deformed configuration $\boldsymbol{\varphi}, \boldsymbol{t}$ is performed in a similar fashion as for $\boldsymbol{\varphi}_{0}, \boldsymbol{T}$

$$
\boldsymbol{u}^{h}\left(\xi^{1}, \xi^{2}\right)=\sum_{a=1}^{n_{e n}} N_{a}\left(\xi^{1}, \xi^{2}\right) \boldsymbol{u}_{a}, \quad \boldsymbol{\varphi}^{h}=\boldsymbol{\varphi}_{0}^{h}+\boldsymbol{u}^{h}, \quad \boldsymbol{t}^{h}\left(\xi^{1}, \xi^{2}\right)=\sum_{a=1}^{n_{e n}} N_{a}\left(\xi^{1}, \xi^{2}\right) \boldsymbol{t}_{a}\left(\boldsymbol{\vartheta}_{a}\right)
$$

The virtual quantities $\delta \boldsymbol{\varphi}$ and $\delta \boldsymbol{t}$ are interpolated in the same manner as $\boldsymbol{\varphi}$ and $\boldsymbol{t}$

$$
\delta \boldsymbol{\varphi}^{h}\left(\xi^{1}, \xi^{2}\right)=\sum_{a=1}^{n_{e n}} N_{a}\left(\xi^{1}, \xi^{2}\right) \delta \boldsymbol{\varphi}_{a}, \quad \delta \boldsymbol{t}^{h}\left(\xi^{1}, \xi^{2}\right)=\sum_{a=1}^{n_{e n}} N_{a}\left(\xi^{1}, \xi^{2}\right) \delta \boldsymbol{t}_{a}
$$

Derivations of $\boldsymbol{\varphi}, \boldsymbol{t}, \delta \boldsymbol{\varphi}$ and $\delta \boldsymbol{t}$ with respect to $\xi^{\alpha}$ coordinates are obtained trivially. To avoid the transverse shear locking, the assumed natural strain (ANS) concept (see [3]) is chosen. The transverse shear strains are evaluated, using (12) and the interpolations (41) and (42), only at element edge mid-points $A, B, C$ and $D$, where $\varphi_{0}^{I}=\frac{1}{2}\left(\varphi_{0 J}+\varphi_{0 K}\right)$ and $I=A, B, C, D$, $J=1,2,3,4$ and $K=2,3,4,1$.

$$
\widetilde{\gamma}_{13}=\frac{1}{2}\left(1-\xi^{2}\right) \widetilde{\gamma}_{13}^{A}+\frac{1}{2}\left(1+\xi^{2}\right) \widetilde{\gamma}_{13}^{C}, \quad \widetilde{\gamma}_{23}=\frac{1}{2}\left(1-\xi^{1}\right) \widetilde{\gamma}_{23}^{D}+\frac{1}{2}\left(1+\xi^{1}\right) \widetilde{\gamma}_{23}^{B}
$$

The transformation to $\widehat{\gamma}_{13}$ and $\widehat{\gamma}_{23}$ is given according to (14).

\section{Computational issues for plasticity}

The solution of the weak form of equilibrium equations (25), discretized in space, is searched for at discrete pseudo-time points $0<t_{1}<\ldots t_{n}<t_{n+1} \ldots<T$. In what follows a pseudotime increment $\Delta t=t_{n+1}-t_{n}$ will be considered. As a result of space discretization, the evolution equations (39) become ordinary differential equations in pseudo-time that are related to each finite element integration point. This enables introduction of operator split method, see e.g. Ibrahimbegovic [14]. This method consists of two sequential (but not independent) solution procedures; one solution procedure (called global) searches for the values of nodal displacements/rotations at pseudo-time instant $t_{n+1}$ at frozen values of internal variables, while the other solution procedure searches for the values of internal variables at integration points at $t_{n+1}$ while keeping frozen nodal displacements/rotations. The later step will be considered in this section. 


\subsection{Equations for computation of internal variables}

For numerical integration of evolution equations (39) the backward Euler integration scheme will be used. At a pseudo-time increment $\Delta t=t_{n+1}-t_{n}$, the pseudo-time integration problem at an integration point located at $\boldsymbol{x}^{h}\left(\xi_{G}^{1}, \xi_{G}^{2}\right) \in A^{h}$ can be stated as: By knowing the values of the internal variables at $t_{n}$, i.e. $\varepsilon_{n}^{p}, \xi_{n}^{I}, \varkappa_{n}$, find, by integrating (39), such values of the internal variables at $t_{n+1}$, i.e. $\varepsilon_{n+1}^{p}, \xi_{n+1}^{I}, \varkappa_{n+1}$, that satisfy the yield criterion. The best guess for the strains at the end of the pseudo-time increment, $\varepsilon_{n+1}^{(i)}$, is given data. Here $(i)$ is an iteration counter of the global Newton-Raphson solution procedure that searches for nodal displacements/rotations at time $t_{n+1}$.

Prior the integration of evolution equations the following test is performed: assume that the pseudo-time step from $t_{n}$ to $t_{n+1}$ remains elastic, evaluate the trial (test) values of strain-like and stress-like internal variables

$$
\boldsymbol{\sigma}_{n+1}^{\text {trial }}=\boldsymbol{C}(\boldsymbol{\varepsilon}_{n+1}^{(i)}-\underbrace{\boldsymbol{\varepsilon}_{n+1}^{p, \text { trial }}}_{\boldsymbol{\varepsilon}_{n}^{p}}), \quad q_{n+1}^{\text {trial }}=-\Xi^{\prime}(\underbrace{\xi_{n+1}^{I, \text { trial }}}_{\xi_{n}^{I}}), \quad \boldsymbol{\alpha}_{n+1}^{\text {trial }}=-\frac{2}{3} H_{k i n} \underbrace{\boldsymbol{\varkappa}_{n+1}^{\text {trial }}}_{\varkappa_{n}},
$$

and the trial values of yield functions $\phi_{\mu, n+1}^{t r}=\phi_{\mu}\left(\boldsymbol{\sigma}_{n+1}^{\text {trial }}, q_{n+1}^{\text {trial }}, \boldsymbol{\alpha}_{n+1}^{\text {trial }}\right)$. Depending on the values of the trial yield functions we distinguish between a) elastic and b) plastic step.

a) Elastic step: If both yield functions are satisfied, i.e. $\phi_{\mu, n+1}^{t r} \leq 0$, then, see $(40), \gamma_{\mu, n+1}=$ $\dot{\gamma}_{\mu} \Delta t=0$. The final values at the end of the pseudo-time increment (marked with the bar) equal the trial values, i.e. $\bar{\varepsilon}_{n+1}^{p}=\varepsilon_{n+1}^{p, \text { trial }}, \bar{\xi}_{n+1}^{I}=\xi_{n+1}^{I, \text { trial }}$ and $\overline{\boldsymbol{\varkappa}}_{n+1}=\boldsymbol{\varkappa}_{n+1}^{\text {trial }}$. The pseudo-time step is indeed elastic.

b) Plastic step: In the case that one or both yield functions for the trial values are violated, then $\gamma_{\mu, n+1}>0$ and $\phi_{\mu, n+1}=0$ for $\mu=1$ or/and $\mu=2$. The backward Euler integration of evolution equations is performed, i.e.

$$
\varepsilon_{n+1}^{p}=\varepsilon_{n}^{p}+\underbrace{\sum_{\mu=1}^{2} \gamma_{\mu, n+1} \nu_{\mu, n+1}}_{\Delta \varepsilon_{n+1}^{p}}, \xi_{n+1}^{I}=\xi_{n}^{I}+\sum_{\mu=1}^{2} \gamma_{\mu, n+1} \beta_{n+1}, \varkappa_{n+1}=\varkappa_{n}+\sum_{\mu=1}^{2} \gamma_{\mu, n+1} \boldsymbol{\nu}_{\mu, n+1}
$$

The final values of parameters $\bar{\varepsilon}_{n+1}^{p}, \bar{\xi}_{n+1}^{I}, \bar{\varkappa}_{n+1}, \bar{\gamma}_{1, n+1}$ and $\bar{\gamma}_{2, n+1}$ are determined by solving equations (46). The solution must be an admissible one. Since we deal with two-surface yield criteria, one of three different sets of constrains must be enforced. First set $\phi_{1, n+1}=0$ and $\phi_{2, n+1}=0$ corresponds to the case when both yield surfaces are active. When only the first surface is active the constraints are $\phi_{1, n+1}=0$ and $\gamma_{2, n+1}=0$ and when only the second surface is active the constraints are $\gamma_{1, n+1}=0$ and $\phi_{2, n+1}=0$. The corresponding procedures will be further addressed in section 3.2.

The rest of this section will be related to the case when both yield surfaces are active. We search for solution of eqs. (46) constrained by $\phi_{1, n+1}=0$ and $\phi_{2, n+1}=0$. There are two options of handling this problem:

(i) The equations (46), $\phi_{1, n+1}=0$ and $\phi_{2, n+1}=0$ are rearranged in such a way that only two equations $\left(\phi_{1, n+1}=0\right.$ and $\left.\phi_{2, n+1}=0\right)$ need to be solved in order to determine the values of internal variables,

(ii) The equations (46), $\phi_{1, n+1}=0$ and $\phi_{2, n+1}=0$ are solved simultaneously. 


\subsubsection{Option (i)}

By using (46), one can express stress resultants at $t_{n+1}$ as

$$
\begin{aligned}
& \boldsymbol{\sigma}_{n+1}=\boldsymbol{C} \underbrace{\left(\boldsymbol{\varepsilon}_{n+1}^{(i)}-\boldsymbol{\varepsilon}_{n+1}^{p}\right)}_{\boldsymbol{\varepsilon}_{n+1}^{(i)}-\boldsymbol{\varepsilon}_{n}^{p}-\Delta \boldsymbol{\varepsilon}_{n+1}^{p}}=\boldsymbol{\sigma}_{n+1}^{\text {trial }}-\sum_{\mu=1}^{2} \gamma_{\mu, n+1} \boldsymbol{C} \boldsymbol{\nu}_{\mu, n+1}, \\
& q_{n+1}=-\boldsymbol{\Xi}^{\prime}\left(\xi_{n}^{I}+\sum_{\mu=1}^{2} \gamma_{\mu, n+1} \beta_{n+1}\right), \\
& \boldsymbol{\alpha}_{n+1}=-\frac{2}{3} H_{k i n} \boldsymbol{\varkappa}_{n+1}=\boldsymbol{\alpha}_{n+1}^{\text {trial }}-\frac{2}{3} H_{k i n} \sum_{\mu=1}^{2} \gamma_{n+1} \boldsymbol{\nu}_{\mu, n+1} .
\end{aligned}
$$

Since $\boldsymbol{\nu}_{\mu, n+1}=2 \boldsymbol{A}_{\mu}\left(\boldsymbol{\sigma}_{n+1}+\boldsymbol{\alpha}_{n+1}\right)$, one can conclude from (47) that

$$
\begin{aligned}
\left(\boldsymbol{\sigma}_{n+1}+\boldsymbol{\alpha}_{n+1}\right) & =\left[\boldsymbol{I}_{8}+\sum_{\mu=1}^{2} \gamma_{\mu, n+1}\left(2 \boldsymbol{C} \boldsymbol{A}_{\mu}+\frac{4}{3} H_{k i n} \boldsymbol{A}_{\mu}\right)\right]^{-1}\left(\boldsymbol{\sigma}_{n+1}^{\text {trial }}+\boldsymbol{\alpha}_{n+1}^{\text {trial }}\right) \\
q_{n+1} & =q_{n+1}\left(\xi_{n}^{I}+\sum_{\mu=1}^{2} \gamma_{\mu, n+1} \beta_{n+1}\right)
\end{aligned}
$$

where the matrix product $\boldsymbol{C} \boldsymbol{A}_{\mu}$ is

$$
\boldsymbol{C A}_{\mu}=\left[\begin{array}{lll}
\frac{1}{n_{0}^{2}} \boldsymbol{C}^{n} \boldsymbol{P} & \frac{\operatorname{sign}(\mu)}{2 \sqrt{3} n_{0} m_{0}} \boldsymbol{C}^{n} \boldsymbol{P} & \mathbf{0} \\
\frac{\operatorname{sign}(\mu)}{2 \sqrt{3} n_{0} m_{0}} \boldsymbol{C}^{b} \boldsymbol{P} & \frac{1}{m_{0}^{2}} \boldsymbol{C}^{b} \boldsymbol{P} & \mathbf{0} \\
\mathbf{0} & \mathbf{0} & \frac{1}{q_{0}^{2}} \boldsymbol{C}^{s} \boldsymbol{I}_{2}
\end{array}\right]
$$

For the linear isotropic hardening case one can obtain from (36), (39) and (49) that

$$
\beta_{n+1}=\frac{2\left(K_{h} \xi_{n}^{I}+\sigma_{y}\right)}{\sigma_{y}^{2}-2 \sum_{\mu=1}^{2} \gamma_{\mu, n+1} K_{h}} .
$$

The inverse in (48) can be obtained explicitly. Since the shear part of $\left(\boldsymbol{\sigma}_{n+1}+\boldsymbol{\alpha}_{n+1}\right)$ is uncoupled from the membrane and bending parts, one can use (48) to get the following expression for the shear stress resultants

$$
\left(\boldsymbol{q}_{n+1}+\boldsymbol{\alpha}_{n+1}^{q}\right)=\frac{1}{\left(1+\left(\gamma_{n+1}^{1}+\gamma_{n+1}^{2}\right) \frac{2}{q_{0}^{2}}\left(k^{s}+\frac{2}{3} H_{k i n}\right)\right)}\left(\boldsymbol{q}_{n+1}^{\text {trial }}+\boldsymbol{\alpha}_{n+1}^{q, \text { trial }}\right) .
$$

$\boldsymbol{P}$ and $\overline{\boldsymbol{C}}$ have the same characteristic subspaces, i.e.

$$
\boldsymbol{P}=\boldsymbol{Q} \boldsymbol{\Lambda}_{\boldsymbol{P}} \boldsymbol{Q}^{T}, \quad \overline{\boldsymbol{C}}=\boldsymbol{Q} \boldsymbol{\Lambda}_{\bar{C}} \boldsymbol{Q}^{T}, \quad \boldsymbol{Q}=\frac{1}{\sqrt{2}}\left[\begin{array}{ccc}
1 & 1 & 0 \\
-1 & 1 & 0 \\
0 & 0 & \sqrt{2}
\end{array}\right]
$$

where $\boldsymbol{Q}^{T}=Q^{-1}$ and

$$
\boldsymbol{\Lambda}_{\boldsymbol{P}}=\left[\begin{array}{ccc}
\frac{1}{2} & 0 & 0 \\
0 & \frac{3}{2} & 0 \\
0 & 0 & 3
\end{array}\right], \quad \boldsymbol{\Lambda}_{\bar{C}}=\left[\begin{array}{ccc}
\frac{E}{1-\nu} & 0 & 0 \\
0 & \frac{E}{1+\nu} & 0 \\
0 & 0 & \frac{E}{2(1+\nu)}
\end{array}\right]
$$


By defining $\hat{\boldsymbol{\sigma}}_{n+1}=\left[\boldsymbol{n}^{T}, \boldsymbol{m}^{T}\right]^{T}, \hat{\boldsymbol{\alpha}}_{n+1}=\left[\boldsymbol{\alpha}^{n, T}, \boldsymbol{\alpha}^{m, T}\right]^{T}$ and by using (51) one can get the following expression from (48)

$$
\begin{aligned}
& \operatorname{diag}\left[\boldsymbol{Q}^{T}, \boldsymbol{Q}^{T}\right]\left(\hat{\boldsymbol{\sigma}}_{n+1}+\hat{\boldsymbol{\alpha}}_{n+1}\right)
\end{aligned}
$$

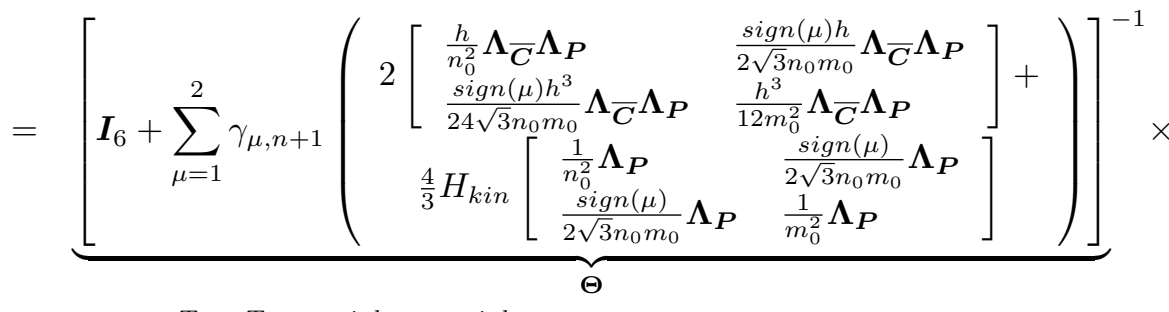

$$
\begin{aligned}
& \operatorname{diag}\left[\boldsymbol{Q}^{T}, \boldsymbol{Q}^{T}\right]\left(\hat{\boldsymbol{\sigma}}_{n+1}^{\text {trial }}+\hat{\boldsymbol{\alpha}}_{n+1}^{\text {trial }}\right),
\end{aligned}
$$

where the matrix $\Theta$ in (52) is of the following form

$$
\boldsymbol{\Theta}=\left[\begin{array}{cccccc}
\widetilde{a} & 0 & 0 & \widetilde{g} & 0 & 0 \\
0 & \widetilde{b} & 0 & 0 & \widetilde{h} & 0 \\
0 & 0 & \widetilde{c} & 0 & 0 & \widetilde{i} \\
\widetilde{j} & 0 & 0 & \widetilde{d} & 0 & 0 \\
0 & \widetilde{k} & 0 & 0 & \widetilde{e} & 0 \\
0 & 0 & \widetilde{l} & 0 & 0 & \widetilde{f}
\end{array}\right],
$$

with

$$
\begin{aligned}
\widetilde{a} & =\frac{-\left(\gamma_{1, n+1}+\gamma_{2, n+1}\right)\left(3 E h-2 H_{k i n}(\nu-1)\right)}{3 n_{0}^{2}(\nu-1)}+1, \widetilde{b}=\frac{\left(\gamma_{1, n+1}+\gamma_{2, n+1}\right)\left(3 E h+2 H_{k i n}(1+\nu)\right)}{n_{0}^{2}(1+\nu)}+1, \\
\widetilde{c} & =\frac{\left(\gamma_{1, n+1}+\gamma_{2, n+1}\right)\left(3 E h+4 H_{k i n}(1+\nu)\right)}{n_{0}^{2}(1+\nu)}+1, \widetilde{d}=\frac{-\left(\gamma_{1, n+1}+\gamma_{2, n+1}\right)\left(E h^{3}-8 H_{k i n}(\nu-1)\right)}{12 m_{0}^{2}(\nu-1)}+1, \\
\widetilde{e} & =\frac{\left(\gamma_{1, n+1}+\gamma_{2, n+1}\right)\left(E h^{3}+8 H_{k i n}(1+\nu)\right)}{4 m_{0}^{2}(1+\nu)}+1, \widetilde{f}=\frac{\left(\gamma_{1, n+1}+\gamma_{2, n+1}\right)\left(E h^{3}+16 H_{k i n}(1+\nu)\right)}{4 m_{0}^{2}(1+\nu)}+1, \\
\widetilde{g} & =\frac{-\left(\gamma_{1, n+1}-\gamma_{2, n+1}\right)\left(3 E h-2 H_{k i n}(\nu-1)\right)}{6 \sqrt{3} m_{0} n_{0}(\nu-1)}, \widetilde{h}=\frac{\left(\gamma_{1, n+1}-\gamma_{2, n+1}\right)\left(3 E h+2 H_{k i n}(1+\nu)\right)}{2 \sqrt{3} m_{0} n_{0}(1+\nu)}, \\
\widetilde{i} & =\frac{\left(\gamma_{1, n+1}-\gamma_{2, n+1}\right)\left(3 E h+4 H_{k i n}(1+\nu)\right)}{2 \sqrt{3} m_{0} n_{0}(1+\nu)}, \widetilde{j}=\frac{-\left(\gamma_{1, n+1}-\gamma_{2, n+1}\right)\left(E h^{3}-8 H_{k i n}(\nu-1)\right)}{24 \sqrt{3} m_{0} n_{0}(\nu-1)}, \\
\widetilde{k} & =\frac{\left(\gamma_{1, n+1}-\gamma_{2, n+1}\right)\left(E h^{3}+8 H_{k i n}(1+\nu)\right)}{8 \sqrt{3} m_{0} n_{0}(1+\nu)}, \widetilde{l}=\frac{\left(\gamma_{1, n+1}-\gamma_{2, n+1}\right)\left(E h^{3}+16 H_{k i n}(1+\nu)\right)}{8 \sqrt{3} m_{0} n_{0}(1+\nu)} .
\end{aligned}
$$

The form (53) allows inversion of $\boldsymbol{\Theta}$ leading to

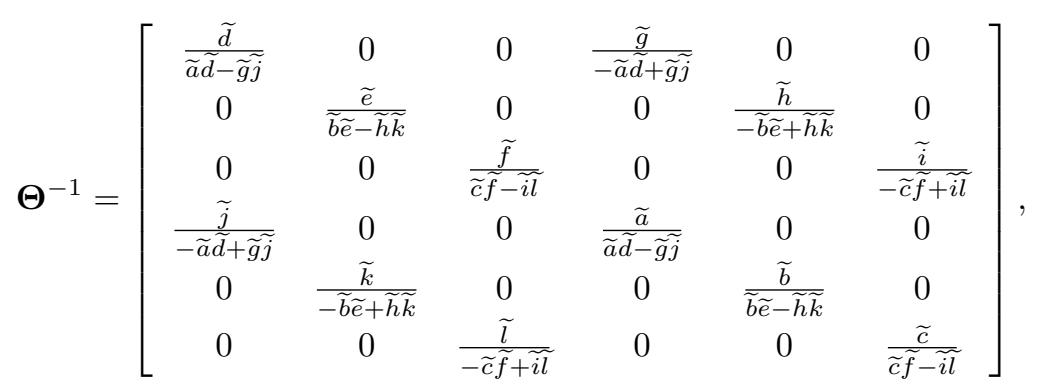

and

$$
\left(\hat{\boldsymbol{\sigma}}_{n+1}+\hat{\boldsymbol{\alpha}}_{n+1}\right)=\operatorname{diag}[\boldsymbol{Q}, \boldsymbol{Q}] \boldsymbol{\Theta}^{-1} \operatorname{diag}\left[\boldsymbol{Q}^{T}, \boldsymbol{Q}^{T}\right]\left(\hat{\boldsymbol{\sigma}}_{n+1}^{\text {trial }}+\hat{\boldsymbol{\alpha}}_{n+1}^{\text {trial }}\right) .
$$


One can now finally write eq. (48) as

$$
\begin{aligned}
& \left(\boldsymbol{\sigma}_{n+1}+\boldsymbol{\alpha}_{n+1}\right)=
\end{aligned}
$$

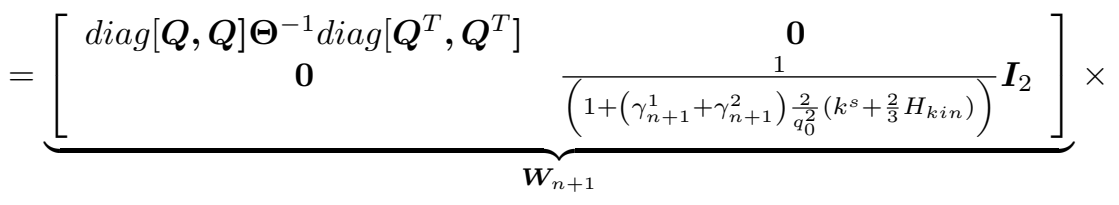

$$
\begin{aligned}
& \left(\boldsymbol{\sigma}_{n+1}^{\text {trial }}+\boldsymbol{\alpha}_{n+1}^{\text {trial}}\right),
\end{aligned}
$$

with $\boldsymbol{Q}$ defined in (51) and $\boldsymbol{\Theta}^{-1}$ explicitly defined in (55). It can be seen that the stress resultants $\left(\boldsymbol{\sigma}_{n+1}+\boldsymbol{\alpha}_{n+1}\right)$ in (56) are only functions of $\gamma_{1, n+1}$ and $\gamma_{2, n+1}$.

Now, the yield functions can be rewritten by using (56) and (49) as

$$
\begin{aligned}
\phi_{\mu}\left(\gamma_{1, n+1}, \gamma_{2, n+1}\right)= & \left(\boldsymbol{\sigma}_{n+1}+\boldsymbol{\alpha}_{n+1}\right)^{T} \boldsymbol{A}_{\mu}\left(\boldsymbol{\sigma}_{n+1}+\boldsymbol{\alpha}_{n+1}\right) \\
& -\left(1-\frac{q_{n+1}\left(\xi_{n}^{I}+\sum_{\mu=1}^{2} \gamma_{\mu, n+1} \beta_{n+1}\right)}{\sigma_{y}}\right)^{2}=0, \quad \mu=1,2,
\end{aligned}
$$

and further as

$$
\begin{aligned}
\phi_{\mu}\left(\gamma_{1, n+1}, \gamma_{2, n+1}\right)= & \left(\boldsymbol{\sigma}_{n+1}^{\text {trial }}+\boldsymbol{\alpha}_{n+1}^{\text {trial }}\right)^{T} \boldsymbol{W}_{n+1}^{T} \boldsymbol{A}_{\mu} \boldsymbol{W}_{n+1}\left(\boldsymbol{\sigma}_{n+1}^{\text {trial }}+\boldsymbol{\alpha}_{n+1}^{\text {trial }}\right) \\
& -\left(1-\frac{q_{n+1}\left(\xi_{n}^{I}+\sum_{\mu=1}^{2} \gamma_{\mu, n+1} \beta_{n+1}\right)}{\sigma_{y}}\right)^{2}=0, \quad \mu=1,2 .
\end{aligned}
$$

One finally gets two equations for $\gamma_{1, n+1}$ and $\gamma_{2, n+1}$,

$$
\boldsymbol{R}_{12,(\mathrm{i})}^{p}\left(\gamma_{1, n+1}, \gamma_{2, n+1}\right)=\left[\begin{array}{l}
\phi_{1}\left(\gamma_{1, n+1}, \gamma_{2, n+1}\right) \\
\phi_{2}\left(\gamma_{1, n+1}, \gamma_{2, n+1}\right)
\end{array}\right]=\mathbf{0},
$$

that are highly nonlinear. Once (59) are solved for converged solutions $\bar{\gamma}_{1, n+1}$ and $\bar{\gamma}_{2, n+1}$, the internal variables (46) and stress-resultants (45) at the end of the time increment can be computed.

\subsubsection{Option (ii)}

We simultaneously solve (46) along with $\phi_{1, n+1}=0$ and $\phi_{2, n+1}=0$ for the unknowns $\varepsilon_{n+1}^{p}, \xi_{n+1}^{I}, \varkappa_{n+1}, \gamma_{1, n+1}$ and $\gamma_{2, n+1}$. We can eliminate $\varkappa_{n+1}$ since $\varkappa_{n+1}=\varepsilon_{n+1}^{p}$. The final system of equations that needs to be solved is then

$$
\boldsymbol{R}_{12,(\mathrm{ii})}^{p}\left(\varepsilon_{n+1}^{p}, \xi_{n+1}^{I}, \gamma_{1, n+1}, \gamma_{2, n+1}\right)=\left[\begin{array}{c}
-\varepsilon_{n+1}^{p}+\boldsymbol{\varepsilon}_{n}^{p}+\sum_{\mu=1}^{2} \gamma_{\mu, n+1} \boldsymbol{\nu}_{\mu, n+1} \\
-\xi_{n+1}^{I}+\xi_{n}^{I}+\sum_{\mu=1}^{2} \gamma_{\mu, n+1} \beta_{n+1} \\
\phi_{1, n+1} \\
\phi_{2, n+1}
\end{array}\right]=\mathbf{0}
$$

where

$$
\begin{aligned}
\phi_{\mu, n+1}= & \left(\boldsymbol{C}\left(\varepsilon_{n+1}^{(i)}-\varepsilon_{n+1}^{p}\right)-\frac{2}{3} H_{k i n} \varkappa_{n+1}\right)^{T} \boldsymbol{A}_{\mu}\left(\boldsymbol{C}\left(\varepsilon_{n+1}^{(i)}-\varepsilon_{n+1}^{p}\right)-\frac{2}{3} H_{k i n} \varkappa_{n+1}\right)(61) \\
& -\left(1-\frac{q_{n+1}\left(\xi_{n+1}^{I}\right)}{\sigma_{y}}\right), \quad \mu=1,2 .
\end{aligned}
$$


The expressions are rather simple. The drawback is that we need to solve a system of 11 equations, thus invert a matrix $11 \times 11$.

Note, that both ways of solving for internal variables, i.e. (59) or (60), should produce the same final result. In the following we will use notation

$$
\boldsymbol{R}_{12}^{p}=\left\{\begin{array}{l}
\boldsymbol{R}_{12,(i)}^{p} \\
\boldsymbol{R}_{12,(i i)}^{p}
\end{array},\right.
$$

when referring to either equations (59) or equations (60).

\subsection{Procedures for choosing active set of yield surface}

In the previous section we have determined the system of equations, (59) or (60), by assuming that both yield surfaces $(\mu=1,2)$ are active, i.e. $\boldsymbol{R}_{12}^{p}=\mathbf{0}$, which is not true in all cases. In the case when only one yield surface is active, equations (59) and (60) are not valid. When only the first surface $(\mu=1)$ is active we replace (59) with

$$
\boldsymbol{R}_{1,(i)}^{p}\left(\gamma_{1, n+1}, \gamma_{2, n+1}\right)=\left[\begin{array}{c}
\phi_{1}\left(\gamma_{1, n+1}, \gamma_{2, n+1}\right) \\
\gamma_{2, n+1}
\end{array}\right]=\mathbf{0}
$$

for option (i) and we replace $\phi_{2, n+1}=0$ with $\gamma_{2, n+1}=0$ in (60) for option (ii) to get $\boldsymbol{R}_{1,(i i)}^{p}$. When only the second surface is active $(\mu=2)$ we use

$$
\boldsymbol{R}_{2,(i)}^{p}\left(\gamma_{1, n+1}, \gamma_{2, n+1}\right)=\left[\begin{array}{c}
\gamma_{1, n+1} \\
\phi_{2}\left(\gamma_{1, n+1}, \gamma_{2, n+1}\right)
\end{array}\right]=\mathbf{0}
$$

instead of (59) for option (i) and we replace $\phi_{1, n+1}=0$ with $\gamma_{1, n+1}=0$ in (60) for option (ii) to get $\boldsymbol{R}_{2,(i i)}^{p}$. In the following we will use the notation

$$
\boldsymbol{R}_{1}^{p}=\left\{\begin{array}{l}
\boldsymbol{R}_{1,(i)}^{p} \\
\boldsymbol{R}_{1,(i i)}^{p}
\end{array} \quad, \quad \boldsymbol{R}_{2}^{p}=\left\{\begin{array}{l}
\boldsymbol{R}_{2,(i)}^{p} \\
\boldsymbol{R}_{2,(i i)}^{p}
\end{array} .\right.\right.
$$

In general, we do not know in advance which of the yield surfaces will be active in the converged state and which of the above sets of equations will produce the right results. Figure 2 depicts three different situations. We can see that for the trial value $\sigma_{n+1}^{\text {trial, }}$ equations (63) give the correct update of the internal variables. Similarly we observe that for $\sigma_{n+1}^{\text {trial,2}}$ we need to solve equations (64). But in a case $\sigma_{n+1}^{t r i a l, 12}$, the right choice of equations is not obvious. Therefore we need to develop a procedure which will automatically choose the right set of equations. Here we address two strategies:

- Procedure 1: We solve all three sets of equations. The admissibility of the solutions is then checked.

- Procedure 2: We change the set of active equations during the iterative process.

A brief description of the procedures 1 and 2 is given below. They are valid for both smaller (option (i)) and larger (option (ii)) sets of equations. 


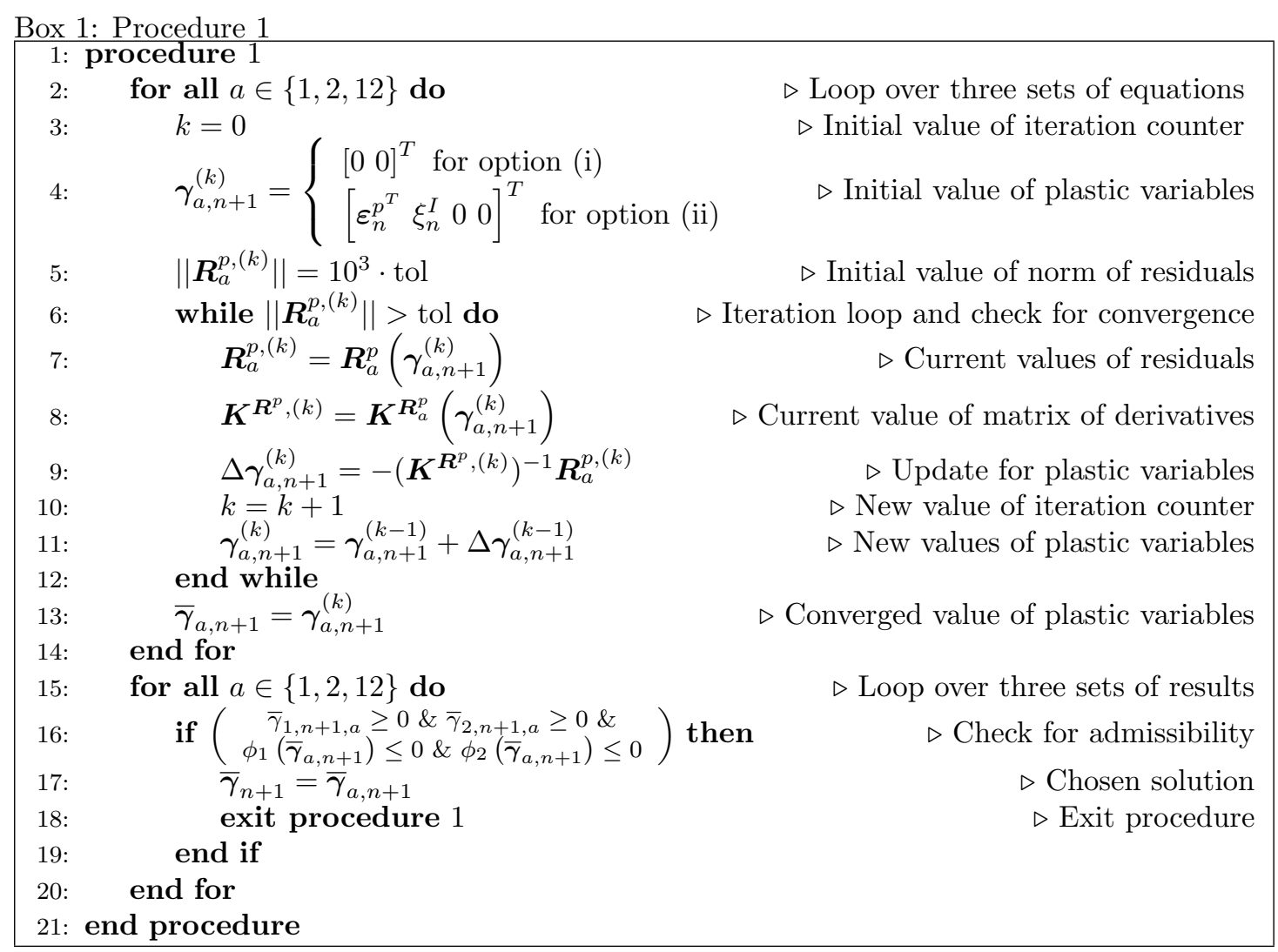




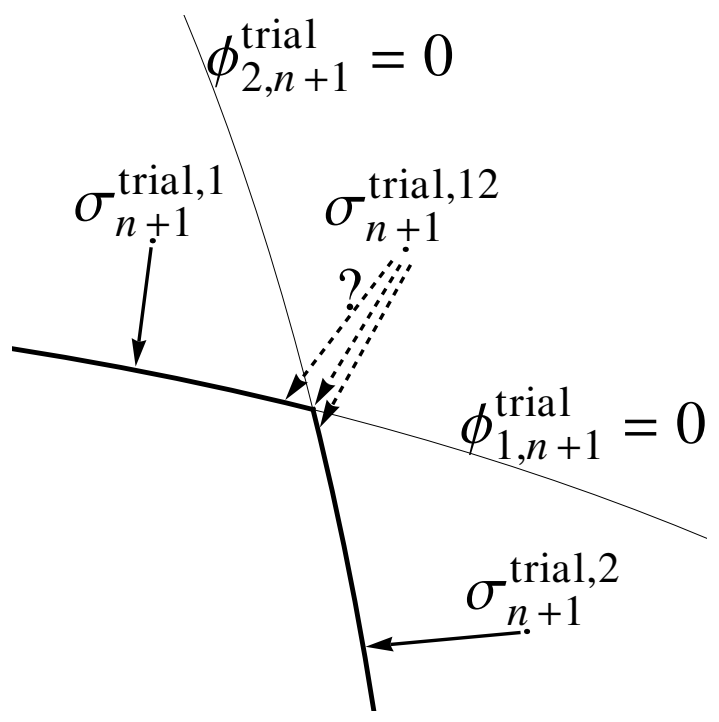

Figure 2: A sketch of a two-surface yield function and the closest point projection

\subsubsection{Procedure 1}

This procedure is presented in Box 1. We solve three different sets of equations

$$
\boldsymbol{R}_{a}^{p}\left(\gamma_{a, n+1}\right)=\mathbf{0} \quad \text { for } \quad a=1,2,12
$$

to get the converged values of

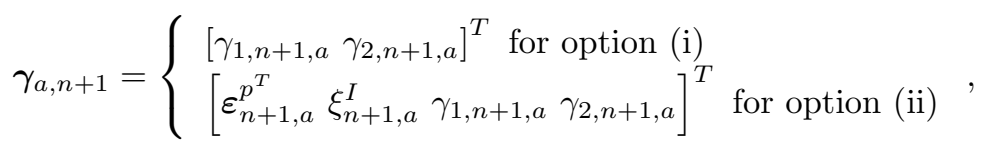

which we denote by $\bar{\gamma}_{a, n+1}$. Each solution is obtained with an iterative procedure with the iteration counter $k$. In the iteration loop we first determine the current values of $\boldsymbol{R}_{a}^{p}$ and compute the current value of the matrix $\boldsymbol{K}^{\boldsymbol{R}_{a}^{p}}=\frac{\partial \boldsymbol{R}_{a}^{p}}{\partial \boldsymbol{\gamma}_{a}}$. Further, we compute the update for plastic variables, set the new value of iteration counter and update the values of the plastic variables. Actions are repeated until convergence criteria is satisfied, i.e. $\left\|\boldsymbol{R}_{a}^{p,(k)}\right\|<$ tol. Once we have the solutions for all three sets of possibly active yield surfaces an additional loop is preformed and the admissibility of each possible solution is checked with the Kuhn-Tucker's loading/unloading conditions. The final solution is the one that satisfies all the conditions.

This procedure is robust and it always provides the solution but it requires computations for three independent (possibly active) sets of yield surfaces, which is computationally demanding.

\subsubsection{Procedure 2}

This procedure is presented in Box 2. It is a variation of a general multi-surface closest point projection iteration procedure, that is presented in [10] and [11]. We first define the iteration counter $k$. In the iteration loop we determine the current values of $\boldsymbol{R}^{p}$ and compute the current value of the matrix $\boldsymbol{K}^{\boldsymbol{R}^{p}}=\frac{\partial \boldsymbol{R}^{p}}{\partial \gamma}$. Further, we compute the update for plastic variables, set the new value of iteration counter and compute the test values of the plastic variables. Next, we 


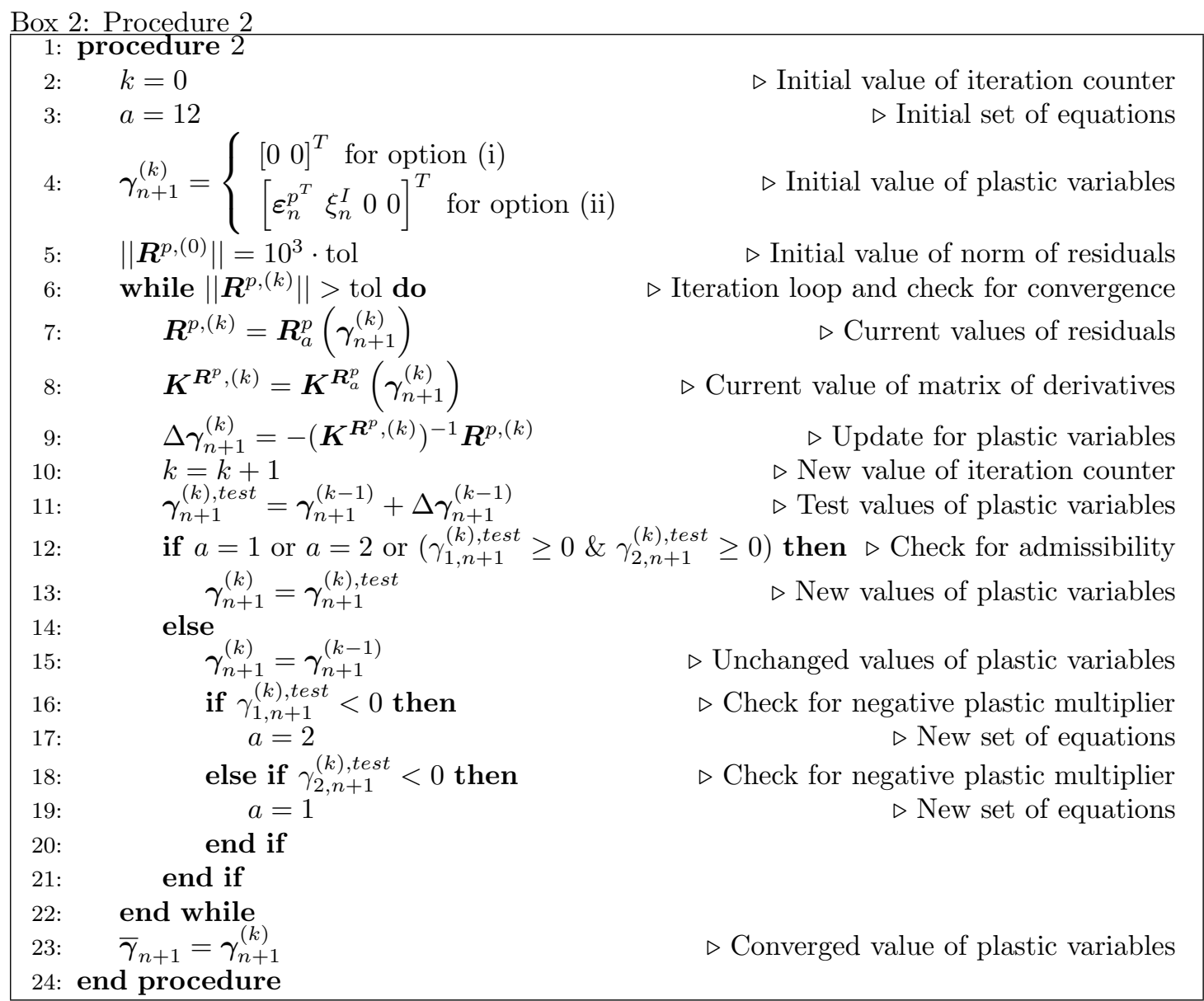


check for admissibility of the test values and if conditions are satisfied we update the values of the plastic variables. If admissibility conditions fail, the plastic variables remain unchanged and we determine the new set of plastic equations by testing for negative values of plastic multipliers. Actions are repeated until convergence criteria is satisfied, i.e. $\left\|\boldsymbol{R}^{p,(k)}\right\|<$ tol.

This procedure is computationally cheaper than procedure 1 , which is obvious, since only the active set of yield surfaces is considered in each iteration $k$. Of course, the change of active set during iterations can slow down the convergence. Procedure 2 is also rather robust for option (i) as shown by numerical examples and also in [10] where similar active set sheme was used. In contrast, numerical examples show that this procedure may choose the wrong active set of yield surfaces for option (ii). The combination of option (ii) and procedure 2 may thus lead to inaccurate results.

\subsubsection{The consistent tangent modulus}

In order to ensure the quadratic rate of convergence of the global iterative procedure we have to consistently linearize the global system of equations (see (27)). This requires to compute the implicit dependencies among the state variables and the strain vector in order to obtain the consistent tangent modulus $\boldsymbol{C}^{e p}=\frac{d \boldsymbol{\sigma}_{n+1}}{d \boldsymbol{\varepsilon}_{n+1}}$. For that purpose we deal with the following functional dependencies

$$
\boldsymbol{\sigma}\left(\varepsilon_{n+1}\right)=\boldsymbol{C}\left(\varepsilon_{n+1}-\varepsilon_{n+1}^{p}\left(\bar{\gamma}_{n+1}\left(\varepsilon_{n+1}\right)\right)\right) .
$$

The challenging part is to obtain the derivatives of the plastic strain with respect to the total strain. By applying the chain rule we have

$$
\frac{d \varepsilon_{n+1}^{p}}{d \varepsilon_{n+1}}=\frac{d \varepsilon_{n+1}^{p}}{d \bar{\gamma}_{n+1}} \frac{d \bar{\gamma}_{n+1}}{d \varepsilon_{n+1}} .
$$

The only unknown derivative here is $\frac{d \overline{\boldsymbol{\gamma}}_{n+1}}{d \boldsymbol{\varepsilon}_{n+1}}$ since $\frac{d \boldsymbol{\varepsilon}_{n+1}^{p}}{d \overline{\boldsymbol{\gamma}}_{n+1}}$ can be easily obtained. The yield surfaces are functions of the total strains and the plastic variables therefore also the $\boldsymbol{R}^{p}$ that is valid $\left(\boldsymbol{R}_{a}^{p}\right.$ at the end of procedure 1 or procedure 2 ) is function of the total strains and the plastic variables

$$
\phi_{\mu, n+1}=\phi_{\mu}\left(\varepsilon_{n+1}, \bar{\gamma}_{n+1}\left(\varepsilon_{n+1}\right)\right) \Rightarrow \boldsymbol{R}^{p}\left(\varepsilon_{n+1}, \bar{\gamma}_{n+1}\left(\varepsilon_{n+1}\right)\right)
$$

The implicit dependencies are obtained from the consistency condition $\dot{\phi}_{\mu, n+1}=0$. By time derivation of $\boldsymbol{R}^{p}$ and by using the chain rule we obtain

$$
\begin{aligned}
\dot{\boldsymbol{R}}_{n+1} & =\frac{d \boldsymbol{R}^{p}}{d \varepsilon_{n+1}} \frac{d \varepsilon_{n+1}}{d t}+\underbrace{\frac{d \boldsymbol{R}^{p}}{d \bar{\gamma}_{n+1}}}_{\boldsymbol{K}^{\boldsymbol{R}^{p}}} \frac{d \bar{\gamma}_{n+1}}{d \varepsilon_{n+1}} \frac{d \varepsilon_{n+1}}{d t}= \\
& =\underbrace{\left(\frac{d \boldsymbol{R}^{p}}{d \varepsilon_{n+1}}+\boldsymbol{K}^{\boldsymbol{R}^{p}} \frac{d \bar{\gamma}_{n+1}}{d \varepsilon_{n+1}}\right)}_{=\mathbf{0}} \underbrace{\frac{d \varepsilon_{n+1}}{d t}}_{\neq \mathbf{0}}=\mathbf{0} .
\end{aligned}
$$

The derivative $\frac{d \overline{\boldsymbol{\gamma}}_{n+1}}{d \boldsymbol{\varepsilon}_{n+1}}$ that we are looking for is then

$$
\frac{d \overline{\boldsymbol{\gamma}}_{n+1}}{d \boldsymbol{\varepsilon}_{n+1}}=-\left(\boldsymbol{K}^{\boldsymbol{R}^{p}}\right)^{-1} \frac{d \boldsymbol{R}^{p}}{d \boldsymbol{\varepsilon}_{n+1}} .
$$

Note that $\boldsymbol{K}^{\boldsymbol{R}^{p}}$ in (72) is computed in both procedures, see Box 1 and Box 2, and that $\frac{d \boldsymbol{R}^{p}}{d \boldsymbol{\varepsilon}_{n+1}}$ can be obtained without difficulties for any $\boldsymbol{R}_{a}^{p}$. 


\section{Numerical examples}

In this section, we present some numerical examples, computed by the 4-node element. By considering notations introduced in 3.1 and 3.2 , the following elements were produced

- elem1i - using procedure 1 (Box 1) and option (i),

- elem1ii - using procedure 1 (Box 1) and option (ii),

- elem2i - using procedure 2 (Box 2) and option (i),

- elem2ii - using procedure 2 (Box 2) and option (ii).

The computer codes were generated by using symbolic code manipulation program AceGen developed by Korelc [9], [8]. The element codes were introduced into the finite element analysis program AceFEM, see Korelc [9].

\subsection{Iso-error maps}

To test the accuracy of the presented algorithms we turn to accuracy analysis by the means of isoerror maps procedure (see [11], [48]). Typically, the iso-error approach is used to test the return mapping algorithm for plane stress elastoplasticity. Here, we adopt this approach to test the return mapping algorithms in a multi-surface plasticity setting. In Figure 3 we present the yield

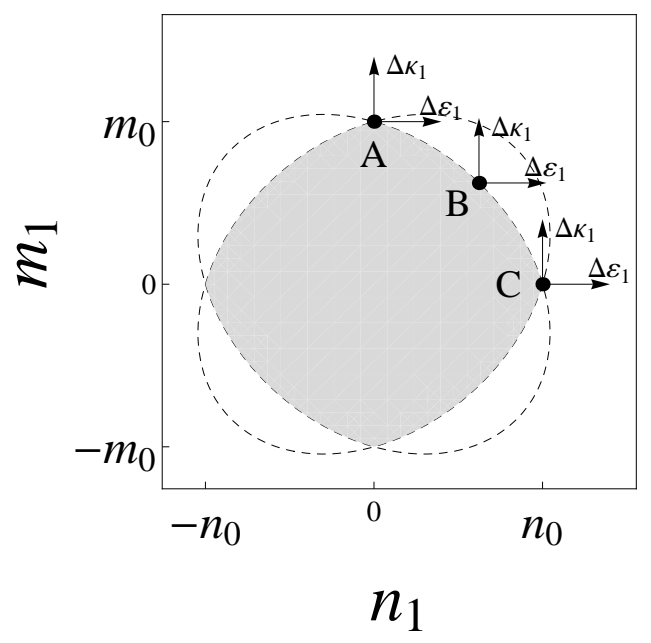

Figure 3: Yield surface with selected points for iso-error maps

surface in the principle axial force principle bending moment plane. The three points in Figure 3 , labeled A, B and C, correspond to pure bending $\left(n_{1}=0, m_{1}=m_{0}\right)$, combination of bending and axial loading $\left(n_{1}=\sqrt{\frac{3}{6+\sqrt{3}}} n_{0}, m_{1}=\sqrt{\frac{3}{6+\sqrt{3}}} m_{0}\right)$ and pure axial loading $\left(n_{1}=n_{0}, m_{1}=0\right)$, respectively. Note that in points $\mathrm{A}$ and $\mathrm{C}$ we have both yield functions active. We construct the iso-error maps with a sequence of specified normalized strain increments, where selected points 
are used as the starting points. The normalization parameters are chosen as the elastic axial strain and elastic curvature associated with initial yielding

$$
\varepsilon_{y}=\frac{\left(1-\nu^{2}\right) \sigma_{y}}{E}, \quad \kappa_{y}=\frac{3\left(1-\nu^{2}\right) \sigma_{y}}{E h} .
$$

By applying the algorithms we then compute the stresses $\hat{\boldsymbol{\sigma}}$. We compare this solution to the "exact" stress $\overline{\boldsymbol{\sigma}}$, which is computed, for any given strain increment, by repeatedly applying the algorithm with increasing number of sub-increments, until the solution converges. The error is obtained according to the expression

$$
\delta=\frac{\sqrt{(\hat{\boldsymbol{\sigma}}-\overline{\boldsymbol{\sigma}})^{T}(\hat{\boldsymbol{\sigma}}-\overline{\boldsymbol{\sigma}})}}{\overline{\boldsymbol{\sigma}}^{T} \overline{\boldsymbol{\sigma}}} \times 100 .
$$

The iso-error maps corresponding to points $\mathrm{A}, \mathrm{B}$ and $\mathrm{C}$ for all here derived algorithms are shown
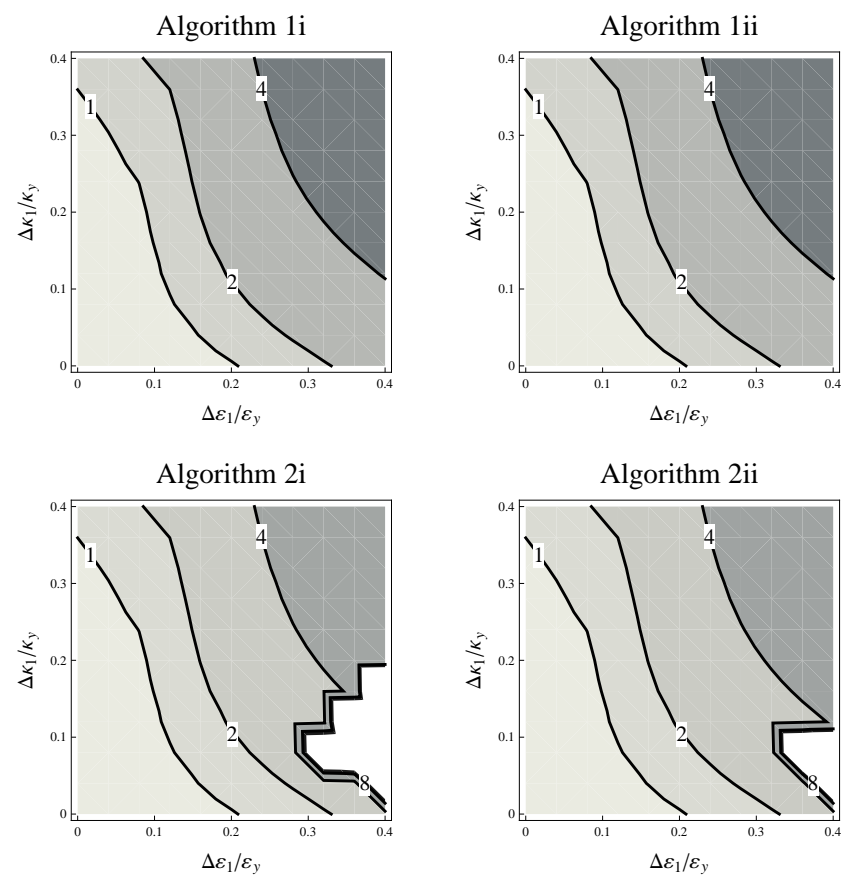

Figure 4: Iso-error maps corresponding to point A

in Figures 4, 5, 6, where we used the parameters: $h=1, E=1, \nu=0, \sigma_{y}=1, K_{h}=0$ and $H_{k i n}=0$. The maps for starting point A (Figure 4) are in perfect agreement in the large portion of the investigated area. The discrepancies appear only in the the lower right regions of maps, corresponding to large increments in axial deformations and small increments in curvatures. Here, the error of algorithms associated with procedure 2 is greater than the error of procedure 1. All the maps for starting point B (Figure 5) are in agreement up to the $1 \%$ iso-error line. Note that the maps associated with procedure 1 are in perfect agreement and that there is also good agreement between the maps associated with procedure 2. The accuracy of the results is therefore dependent on the procedure used, whereas the size of system of plastic equations has very little influence. Again we see that procedure 2 is much more sensitive to the size of strain 

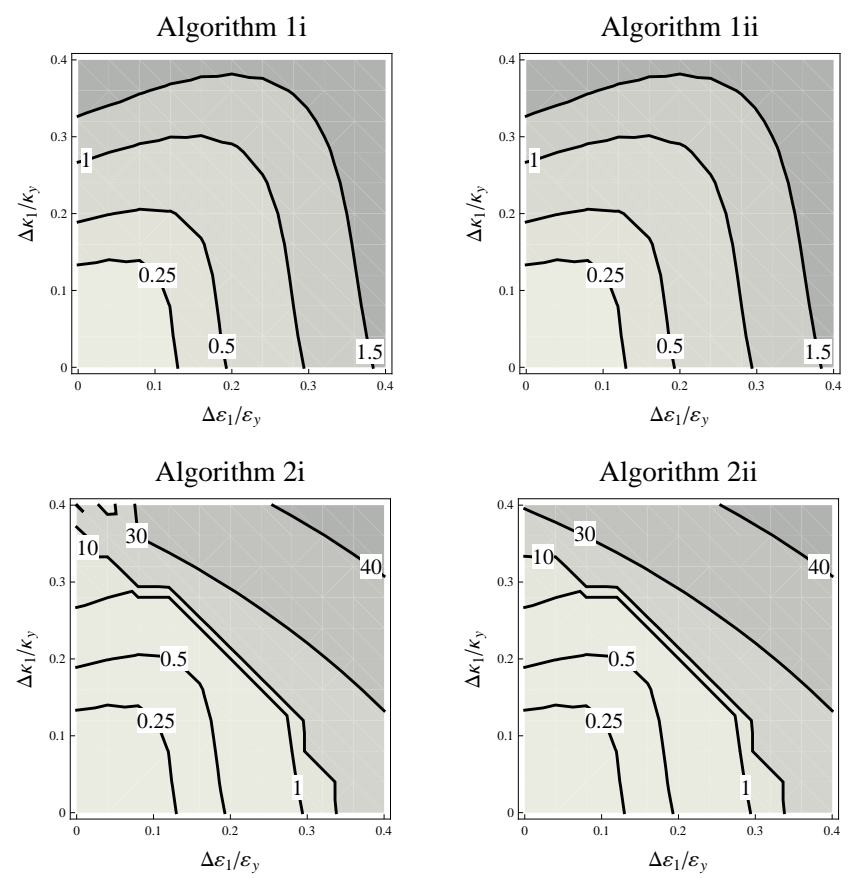

Figure 5: Iso-error maps corresponding to point B
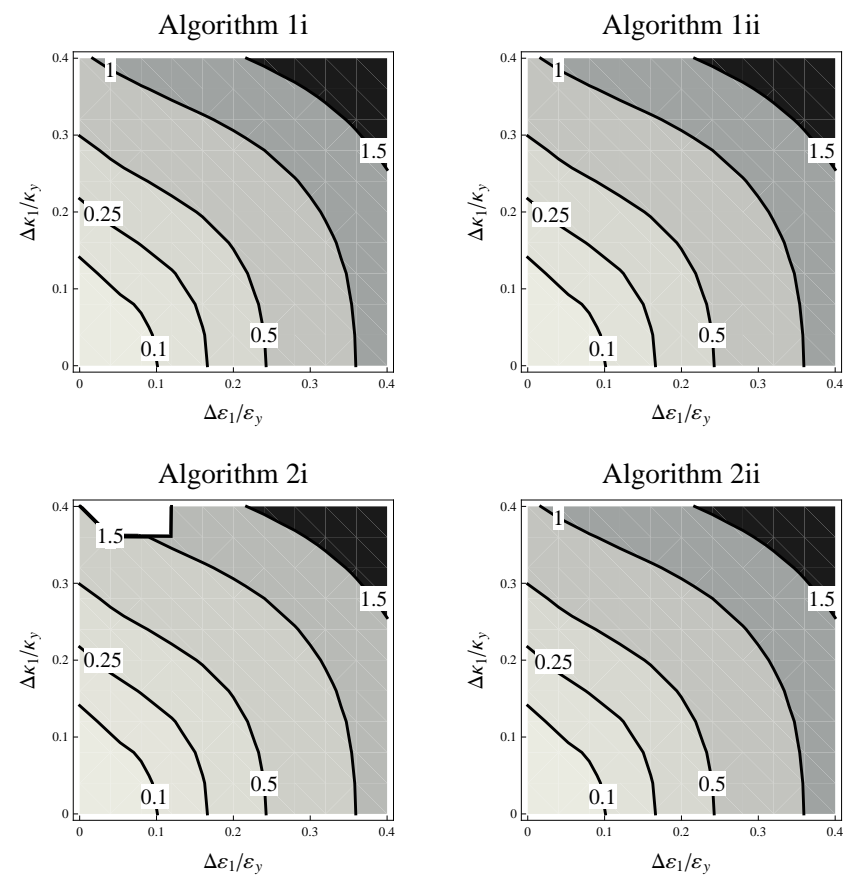

Figure 6: Iso-error maps corresponding to point $\mathrm{C}$ 
increment than procedure 1. In Figure 6 we plot the iso-error maps for point C. There is good agreement between the algorithms except for the distortion which appears in the upper left part of map associated with algorithm $2 \mathrm{i}$.

\subsection{Stretching of cylinder with free ends}

This example is chosen to show that the geometrically nonlinear elastic response of our shell finite element compares well with other representative shell elements. A cylinder with free ends and radius $R=4.953$, length $L=10.35$, thickness 0.094 , elastic modulus $E=10.5 \times 10^{3}$ and Poisson's ratio $\nu=0.3125$ is stretched by two forces $F$ acting in the opposite direction in the middle of the cylinder. Due to the symmetry, only one octant of the cylinder is modeled with appropriate boundary conditions, Fig. 7. The elastic analysis is performed with a mesh of $32 \times 32$

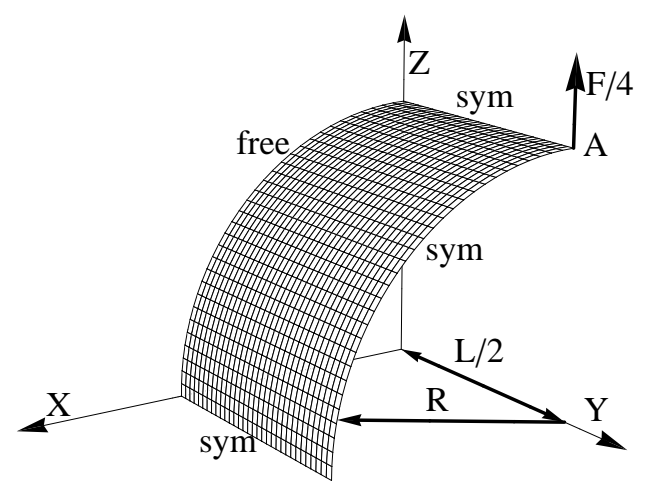

Figure 7: Geometry, loading and boundary conditions for stretched cylinder

elements. For point $A$, the $F / F_{0}\left(F_{0}=50\right)$ versus the $u_{Z}$ displacement curve is presented in Fig. 8 along with the results from Sze et al. [46] and Brank et al. [40]. In [46], a mesh of $16 \times 24$ Abaqus' S4R elements was used. In [40], a mesh of $16 \times 16$ 4-node elements was used. Element in [40] is different from the present one only in the description of finite rotations. It can be seen from Fig. 8 that the shell goes from the bending dominated phase, where the response curve is gently rising, to the membrane dominated phase, where the response curve is steep. At some load level buckling occurs, which is captured slightly differently by the three elements. Nevertheless, our results agree well with the compared ones, which indicates that the present formulation works correctly for nonlinear elastic cases. Initial and deformed configuration (at $u_{Z}=3$ ) are shown in Fig. 9.

\subsection{Stretching of perforated plate}

This example illustrates performances of the derived algorithms for pure membrane state. We consider a plate of length $L=36$, width $B=20$ and thickness $h=1$ that has a hole with radius $R=5$ at its center, Fig. 10. The material data is: elastic modulus $E=70$, Poisson's ratio $\nu=0$ and yield stress $\sigma_{y}=0.243$, while isotropic hardening modulus $K_{h}$ and kinematic hardening modulus $H_{k i n}$ vary. The plate is stretched by prescribed displacements at the two ends. Due to the symmetry, one quadrant of the plate is modeled with the appropriate boundary conditions. 


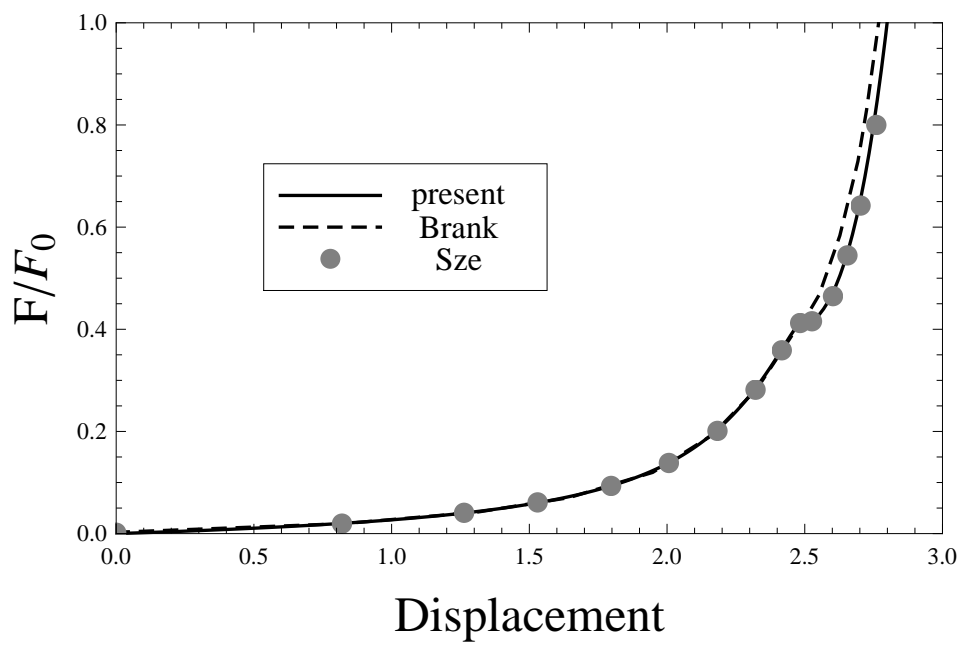

Figure 8: Load versus displacement curves for stretched cylinder

The analyses were geometrically linear. The reaction force versus imposed displacement curves are presented in Fig. 11 for different values of hardening parameters.

The results of the four algorithms are exactly the same for all analyses. In fact, the present stress resultant two-surface plasticity formulation becomes identical to the plane stress von Mises formulation. For no hardening case the computed limit reaction force for a mesh of 96 elements equals to 2.486 , which is almost exact plastic limit force $\sigma_{y}(B-2 R) h=2.43$ if we consider a uniaxial stress state. For $K_{h}=0.2$, our results are identical to the plane stress von Mises plasticity formulation of Fuschi et al. [48], who used equivalent plastic strain as the variable for isotropic hardening and mesh of 72 plane stress elements. In Fig. 11 we also show the response of the plate for one cycle of imposed displacements by using the kinematic hardening.

\subsection{Rectangular plate}

A clamped (of hard type) rectangular plate of elastic-perfectly plastic material under uniformly distributed load in the $Z$ direction is considered. The geometry and material parameters are: length $a=150 \mathrm{~cm}$, width $b=100 \mathrm{~cm}$, thickness $h=5 \mathrm{~cm}$, Young's modulus $E=21000 \mathrm{kN} / \mathrm{cm}^{2}$, Poisson's ration $\nu=0.3$ and yield stress $\sigma_{y}=40 \mathrm{kN} / \mathrm{cm}^{2}$. The geometry and the finite element mesh of the plate are presented in Figure 12. In Figure 13, we plot the load versus central displacement curves of our simulations along with the curve from Dujc [49], where a stress resultant geometrically linear elastoplastic plate formulation was used. We can see that all the algorithms produce the same results and that the shell formulation response is stiffer from the plate formulation due to the membrane stresses. In Figure 14 we plot number of iterations versus time step curves. In this case even the number of iterations in each load step is completely the same for all the algorithms. There is a difference in computational time needed to obtain results though. The computational time for elem $1 i$ is $t_{1 i}=24.0 \mathrm{sec}$, for elem1ii is $t_{1 i i}=10.7 \mathrm{sec}$, for elem2i is $t_{2 i}=26.4 \mathrm{sec}$ and for elem2ii is $t_{2 i i}=9.8 \mathrm{sec}$. We can see that option (ii) algorithms are around two times faster than the option (i) algorithms $\left(t_{1 i}>t_{1 i i}\right.$ and $\left.t_{2 i}>t_{2 i i}\right)$. The choice of procedure has much lower influence on computational time. By comparing slower elements 

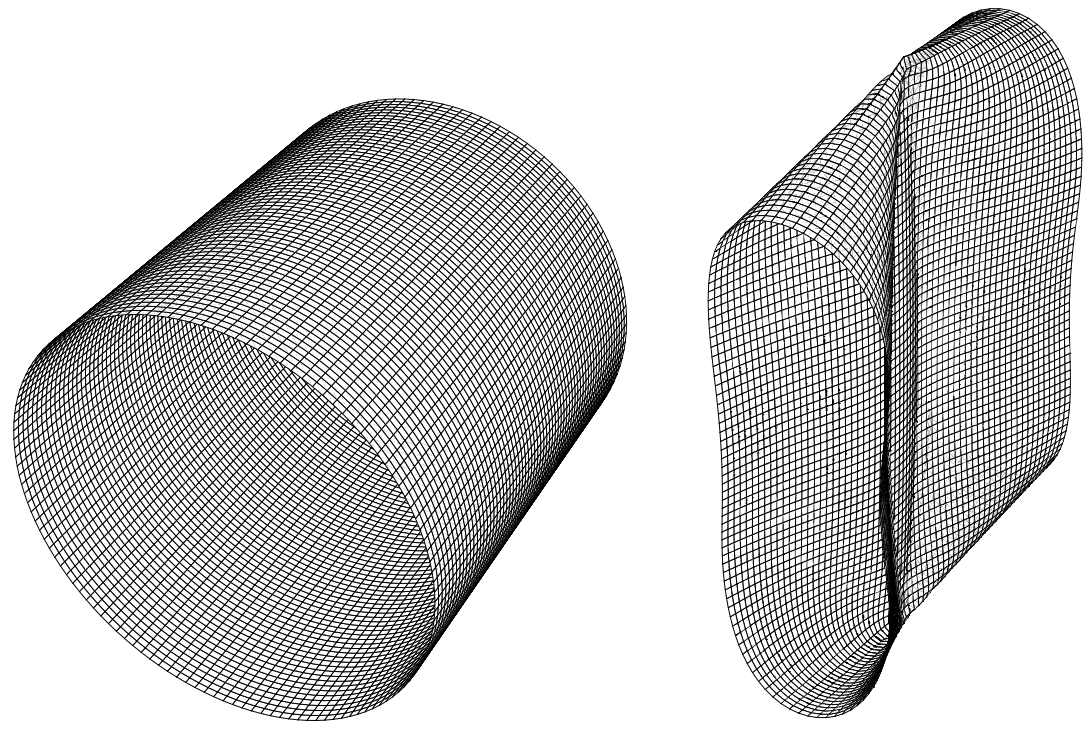

Figure 9: Initial (left) and deformed (right) configuration (at $u_{Z}=3$ ) for stretched cylinder

we see that procedure 1 is faster $\left(t_{1 i}<t_{2 i}\right)$, while in the case of faster elements procedure 2 is faster $\left(t_{1 i i}>t_{2 i i}\right)$.

\subsection{Half of a sphere}

We consider half of a sphere loaded with two inward and two outward forces. Due to the symmetry, only one octant of sphere is modeled. The geometry, loading, boundary conditions and the finite element mesh of the model are presented in Figure 15; $b c_{1}$ and $b c_{2}$ denote the edges with the symmetrical boundary conditions and the edge $b c_{3}$ is free. The radius of the sphere is $10 \mathrm{~cm}$ and the thickness is $0.5 \mathrm{~cm}$. The material properties are: Young's modulus $E=10 \mathrm{kN} / \mathrm{cm}^{2}$, Poisson's ration $\nu=0.2$, yield stress $\sigma_{y}=0.2 \mathrm{kN} / \mathrm{cm}^{2}$ and the linear hardening modulus $K_{h}=3 \mathrm{kN} / \mathrm{cm}^{2}$. Loading was applied in 72 equally spaced time-steps, which was followed by unloading using another 72 time-steps.

The load versus displacement curves of our simulations along with the curves from Simo and Kennedy [10] and Başar and Itskov [2] are presented in Figure 16. Note that in [10] the hardening model was defined as

$$
\begin{aligned}
\phi_{\mu}\left(\boldsymbol{\sigma}, p_{[10]}, \boldsymbol{\alpha}\right) & =(\boldsymbol{\sigma}+\boldsymbol{\alpha})^{T} \boldsymbol{A}_{\mu}(\boldsymbol{\sigma}+\boldsymbol{\alpha})-\left(\frac{\kappa_{0,[10]}+\kappa_{[10]}^{\prime} p_{[10]}}{\kappa_{0,[10]}}\right)^{2}, \\
p_{[10]} & =-\frac{\kappa_{0,[10]}}{\kappa_{[10]}^{\prime}} \alpha_{[10]}, \quad \dot{\alpha}_{[10]}=-\sum_{\mu=1}^{2} \dot{\gamma}_{\mu} \frac{2 \kappa_{[10]}^{\prime}\left(\kappa_{0,[10]}+\kappa_{[10]}^{\prime} p_{[10]}\right)}{\kappa_{0,[10]}^{2}},
\end{aligned}
$$

where $p_{[10]}$ and $\alpha_{[10]}$ are plastic hardening variables and $\kappa_{0,[10]}=0.2 \mathrm{kN} / \mathrm{cm}^{2}$ and $\kappa_{[10]}^{\prime}=9.0$ 


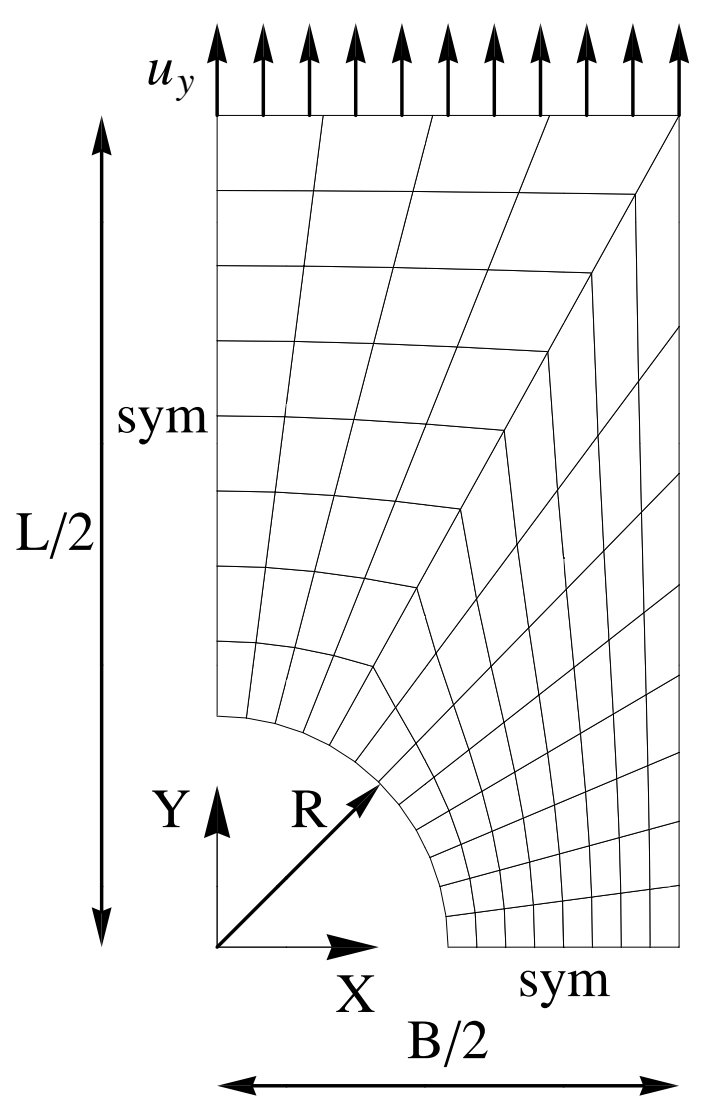

Figure 10: Perforated plate: mesh and boundary conditions

are material parameters. By comparing (31), (36) and (39) with (75) one can, after a short derivation, see that our hardening model is equivalent to model in [10], if we apply the following mapping

$$
\sigma_{y}=\kappa_{0,[10]}=0.2 \mathrm{kN} / \mathrm{cm}^{2}, \quad K_{h}=\kappa_{[10]}^{\prime} \kappa_{0,[10]}=1.8 \mathrm{kN} / \mathrm{cm}^{2}
$$

At load levels below $20 \mathrm{~N}$ all the derived algorithms give the same response, while at higher levels there is a difference in results between elem2ii and the others. We have observed that elem2ii occasionally updates the plastic variables by solving the improper set of equations. The reason is that in procedure 2 we check the admissibility condition in a non-converged state, which in combination with the full system of plastic equations (option (ii)) may lead to an inadmissible solution. This is a significant problem, which leads to the conclusion that the algorithm elem2ii is inaccurate. The observed computational times for this example are: $t_{1 i}=83.6 \mathrm{sec}, t_{1 i i}=42.0 \mathrm{sec}$, $t_{2 i}=117.8 \mathrm{sec}$ and $t_{2 i i}=44.5 \mathrm{sec}$. Similarly to the previous example elem1ii and elem2ii are around two times faster than elem $1 i$ and elem2 $i$. The procedure 1 is faster when comparing slower algorithms $\left(t_{1 i}<t_{2 i}\right)$, which is also the case of faster algorithms $\left(t_{1 i i}<t_{2 i i}\right)$ contrary, to the previous example.

It can be seen from Fig. 16 that algorithms elem $1 i$, elem $1 i i$ and elem $2 i$ are in perfect agreement with the results from Simo and Kennedy [10] for hardening modulus $K_{h}=1.8 \mathrm{kN} / \mathrm{cm}^{2}$. However, our results show discrepancies for larger load values and for the unloading with the 


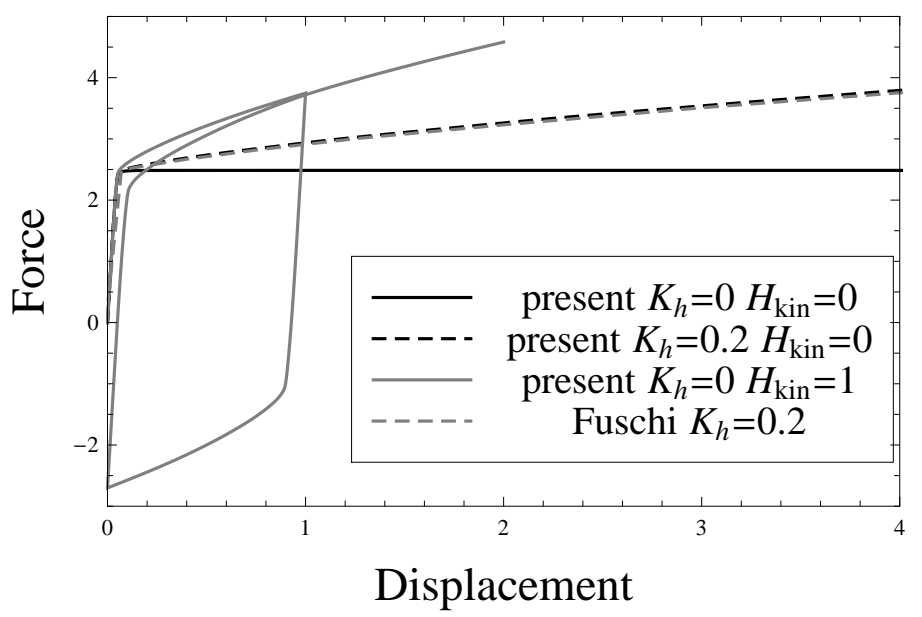

Figure 11: Load versus displacement curves for perforated plate

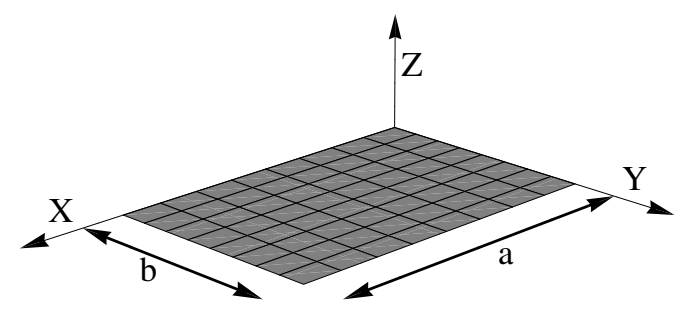

Figure 12: Geometry for rectangular plate

results from Başar and Itskov [2], who used 3d large strain plasticity formulation and solid-shell element. The stress resultant plasticity element is more flexible than the stress-based plasticity. This can be related to the inability of the former element to treat the shell cross-section as partly elastic and partly plastic. At larger load values, where plastification is considerable, many cross-sections obviously remain elastic in the core, which cannot be captured by the stress resultant plasticity. This may explain stiffer response of the stress-based formulation. At Fig. 17 two deformed configurations are presented along with the values of the hardening parameter $\xi^{I}$.

\subsection{Pinched cylinder with isotropic hardening}

We consider a short cylinder bounded by two rigid diaphragms at its ends. The cylinder is loaded with two concentrated forces at the middle section. Due to the symmetry, only one octant of the cylinder is modeled. The geometry, loading and boundary conditions of the octant are presented in Figure 18, where $a=300 \mathrm{~cm}, b c_{1}$ denotes the edge with the rigid diaphragm, while $b c_{2}, b c_{3}$ and $b c_{4}$ denote the edges with the symmetry boundary conditions. The thickness of the cylinder wall is $3 \mathrm{~cm}$ and the material properties are: Young's modulus $E=3000 \mathrm{kN} / \mathrm{cm}^{2}$, Poisson's ration 


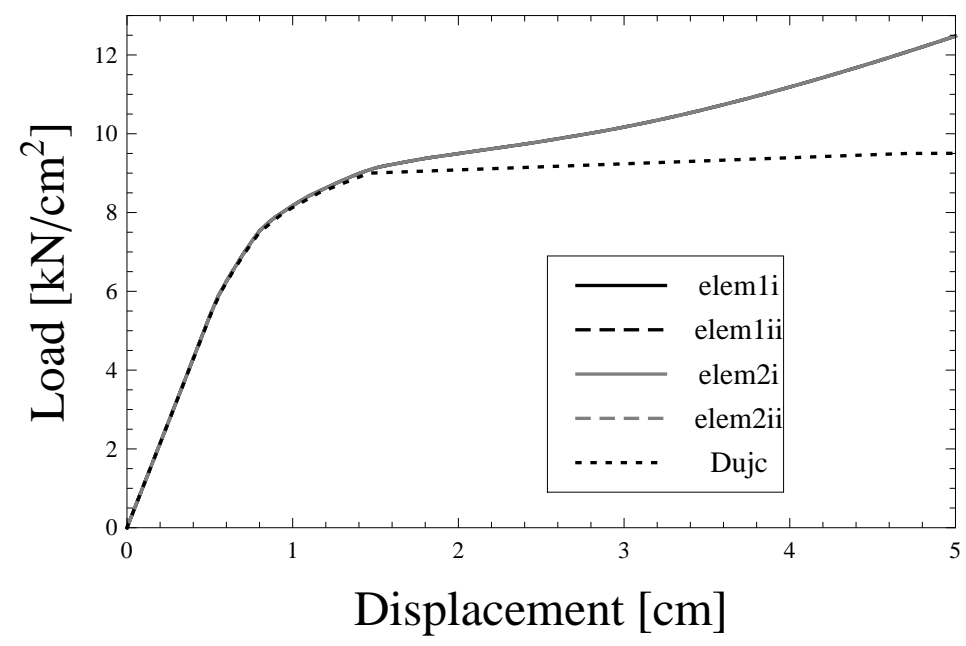

Figure 13: Load versus displacement curves of the thick plate

$\nu=0.3$ and yield stress $\sigma_{y}=24.3 \mathrm{kN} / \mathrm{cm}^{2}$. The plastic behavior is characterized by linear isotropic hardening response with hardening modulus $K_{h}=300 \mathrm{kN} / \mathrm{cm}^{2}$. The load versus displacement curves of our simulations, obtained by meshes of $16 \times 16$ and $32 \times 32$ elements, along with the curves obtained in Simo and Kennedy [10] and Brank et al. [4] are presented in Figure 19. There is a perfect agreement in results for elem1i, elem1ii and elem2i whereas elem2ii, similarly as in the previous example, gives a different solution. The observed computational times for coarser mesh for the first 200 loading steps (equivalent to imposed displacement $100 \mathrm{~cm}$ ) are: $t_{1 i}=203.5 \mathrm{sec}, t_{1 i i}=150.5 \mathrm{sec}, t_{2 i}=211.0 \mathrm{sec}$ and $t_{2 i i}=165.9 \mathrm{sec}$. Algorithms with full system of plastic equations (elem1ii and elem2ii) are faster than the algorithms with the reduced system of plastic equations (elem1i and elem2i) and procedure 1 is faster than procedure $2\left(t_{1 i}<t_{2 i}\right.$ and $\left.t_{1 i i}<t_{2 i i}\right)$. Fig. 19 shows discrepancies of our results, with the results of [10] and [4]. Comparison between the mesh of $32 \times 32$ elements and the mesh of $16 \times 16$ elements shows that the coarser mesh snap-throughs are artificial. They appear when the buckles move through the mesh [50]. In [4], a mesh of $32 \times 32$ stress-based von Mises elements was used with the equivalent plastic strain as the hardening variable and the value of hardening modulus was 50 . The isotropic hardening models and material parameters presented here and in [4] are therefore different, which causes the discrepancies in results. In [10], the mesh of $32 \times 32$ stress resultant plasticity elements was used, with the hardening response defined in (75) and material parameters $\kappa_{0,[10]}=24.3 \mathrm{kN} / \mathrm{cm}^{2}$ and $\kappa_{[10]}^{\prime}=300$. In order to make our hardening model equivalent to model of [10], we need to modify the material parameters to $\sigma_{y}=\kappa_{0,[10]}=0.2 \mathrm{kN} / \mathrm{cm}^{2}$ and $K_{h}=\kappa_{[10]}^{\prime} \kappa_{0,[10]}=7290 \mathrm{kN} / \mathrm{cm}^{2}$. In Fig. 20, our results (mesh is $32 \times 32$ ) for different values of $K_{h}$ are presented along with results obtained in [10] and [4]. Our results for hardening modulus $K_{h}=50 \mathrm{kN} / \mathrm{cm}^{2}$ are now in better agreement with those from [4]. Note, that our results for the modified value of hardening modulus $K_{h}=7290 \mathrm{kN} / \mathrm{cm}^{2}$ are now in good agreement with those from [10].

Finally, Fig. 21 compares our results with a computation where the transverse shear effects were treated as completely elastic. It can be seen that the results are practically identical, which confirms that for this example $(h / a=0.01)$ the plastic transverse shear effects are negligible. 


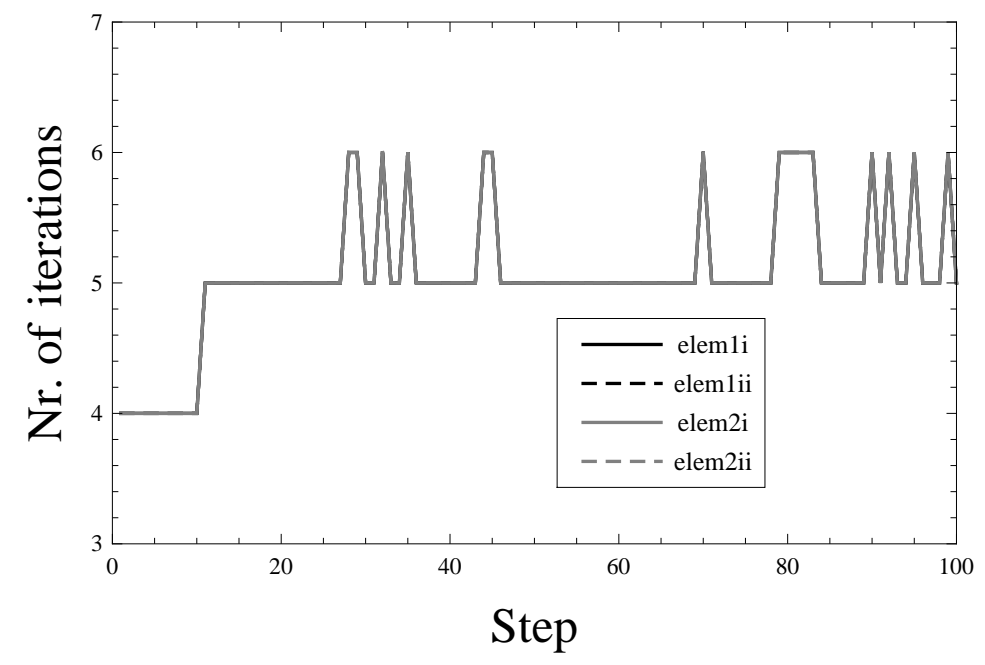

Figure 14: Number of iterations versus time step curves

\subsection{Cylinder under shear load}

We consider a cylinder subjected to shear. The cylinder is clamped at the bottom and at the top it is subjected to imposed displacement in the $\mathrm{Y}$ direction, while the remaining degrees of freedom are set to zero. The geometry and the finite element mesh composed of $64 \times 36$ elements are presented in Figure 23, where $R=28.5 \mathrm{~cm}, H=85 \mathrm{~cm}$ and the thickness of the cylinder is $0.5 \mathrm{~cm}$. Material properties are: Young's modulus $E=21000 \mathrm{kN} / \mathrm{cm}^{2}$, Poisson's ration $\nu=0.3$, yield stress $\sigma_{y}=24.0 \mathrm{kN} / \mathrm{cm}^{2}$ and $K_{h}=0 \mathrm{kN} / \mathrm{cm}^{2}$ thus giving perfectly plastic response. The reaction force in the $\mathrm{Y}$ direction versus imposed displacement curves of our formulations along with the curve obtained by ABAQUS [1] by using the same mesh (the S4R finite element with reduced integration, stress-strain constitutive law and 7 integration points in the thickness direction) are presented in Figure 24. One can see that despite a difference in the finite element formulations the responses are similar.

In this example the results of all in here derived algorithms are in agreement. The observed computational times of our analyses for 15 elastic and 15 inelastic loading steps (equivalent to imposed displacement $0.3 \mathrm{~cm}$ ) are $t_{1 i}=115.9 \mathrm{sec}, t_{1 i i}=74.1 \mathrm{sec}, t_{2 i}=99.1 \mathrm{sec}$ and $t_{2 i i}=72.0 \mathrm{sec}$ and the observed time by using ABAQUS was 26.3 sec. The commercial program is around three times faster than our fastest algorithm, however it is hard to know if the reason is plastic update, element formulation or program structure. Again elements with full system of plastic equations are faster than the elements with the reduced system of plastic equations. In this example procedure 2 was faster than procedure 1. In Figure 25 the deformed configurations at $u_{Y}=10 \mathrm{~cm}$ with the pattern of equivalent plastic strain obtained by ABAQUS [1] and by our elements are presented. Note that the patterns are very similar.

\subsection{Scordelis-Lo roof}

A failure analysis of the Scordelis-Lo roof is considered in this example. The geometry is presented in Figure 26, where $R=7.6 \mathrm{~m}, L=7.6 \mathrm{~m}, \theta=40^{\circ}$ and the thickness is $0.076 \mathrm{~m}$. Material 


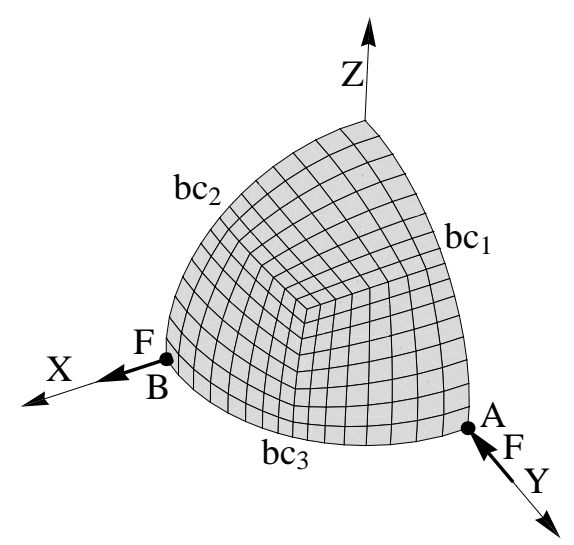

Figure 15: Geometry, loading and boundary conditions of half of a sphere

properties are: Young's modulus $E=2.1 \times 10^{7} \mathrm{kN} / \mathrm{m}^{2}$, Poisson's ration $\nu=0.0$, yield stress $\sigma_{y}=4200 \mathrm{kN} / \mathrm{m}^{2}$ and $K_{h}=0 \mathrm{kN} / \mathrm{m}^{2}$. The roof is submitted to gravity load with the reference self-weight value of $f_{0}=4.0 \mathrm{kN} / \mathrm{m}^{2}$. Due to the symmetry only one quarter of the roof (darkened area in Figure 26) is considered, where we assumed symmetry conditions along lines $X_{1}=0$ and $X_{2}=0$. The response of one quarter of the roof was obtained by two different meshes of $8 \times 8$ and $32 \times 32$ elements and by employing standard arc-length method. In Figure 27 we plot our load versus displacement curves along the curves obtained by Brank et al. [4] and Skallerud and Haugen [18]. In Figure 28 we present the deformed configuration (at displacement equal to 2.5 m) obtained by the finer mesh.

All our algorithms in this example produce the same results. By using the coarse mesh we observed the following computational times for the first 290 loading steps (equivalent to load factor 1.45): $t_{1 i}=35.1 \mathrm{sec}, t_{1 i i}=20.4 \mathrm{sec}, t_{2 i}=31.6 \mathrm{sec}$ and $t_{2 i i}=23.7 \mathrm{sec}$. Again we see that solving the full system of plastic equations is faster than solving the reduced one. Here procedure 1 was faster when solving the full system of plastic equations $\left(t_{1 i i}<t_{2 i i}\right)$ and procedure 2 was faster when solving the reduced one $\left(t_{1 i}>t_{2 i}\right)$.

The difference in Fig. 27 between the results from [4] and the present ones for the mesh $32 \times 32$ should come only from the difference in the constitutive models, since the meshes are the same for both cases and the present finite element formulation is only slightly different from the one in [4]. The computed maximum loads are very close.

\section{$5 \quad$ Concluding remarks}

An inelastic geometrically exact shell finite element formulation has been presented with the constitutive model formulated entirely in terms of stress resultants and stress resultant internal variables. The chosen constitutive model was Ilyushin-Shapiro two-surface elastoplasticity with both isotropic and kinematic hardening.

Several return mapping algorithms that can be used for two-surface plasticity have been pre- 


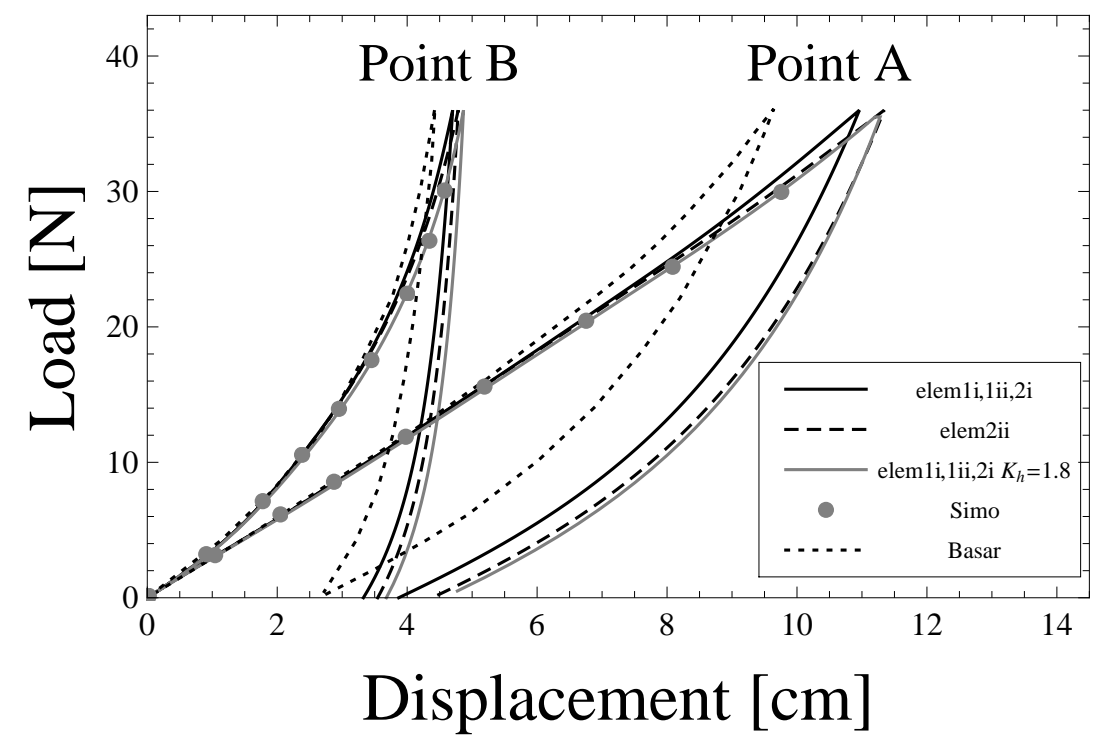

Figure 16: Load versus displacement curves for half of a sphere

sented. These algorithms are combinations of two options and two procedures. The first option (option (i)) reduces the complete set of nonlinear algebraic equations into a single nonlinear scalar equation for each yield surface. The second option (option (ii)) solves the complete set of nonlinear algebraic equations related to the update of internal variables. The two procedures differ from one another by how the right set of yield surfaces is chosen. The first procedure solves the equations of all three possible active sets and concludes on the right set on the basis of final solutions. The second procedure changes the active set of equations during the return mapping iterations. Three the most important findings related to the presented algorithms are: (1) it is faster to solve the complete system of equations (elem $1 i i$, elem $2 i i$ ) than the reduced one (elem $1 i$, elem $2 i),(2)$ the simple and the most straightforward procedure (elem1i, elem $1 i i)$ is equally fast or faster than the active set procedure (elem $2 i$, elem $2 i i),(3)$ the algorithm elem $2 i i$ sometimes chooses the wrong active set of equations in the iterative return mapping process, which is a significant problem, and therefore this procedure cannot be regarded as accurate.

We note that the presented algorithms are not restricted to two-surface Ilyushin-Shapiro plasticity. For example, the elem $1 i$ can be used for a general form of two-surface plasticity. Moreover, in the case of single yield surface, the procedures 1 and 2 would be the same and the four algorithms would converge to two, with one of them solving the complete set of nonlinear algebraic equations, and the other one solving a single nonlinear algebraic equation.

Regarding the stress resultant plasticity, we observed the following. The stress resultant inelastic elements sometimes behave at larger load levels more flexible than the stress-based inelastic elements with through-the-thickness integration, which is result of inability of the stress resultant formulation to treat the cross-section as partly elastic and partly plastic. The transverse shear effects in thin metal shells can be treated as elastic. 

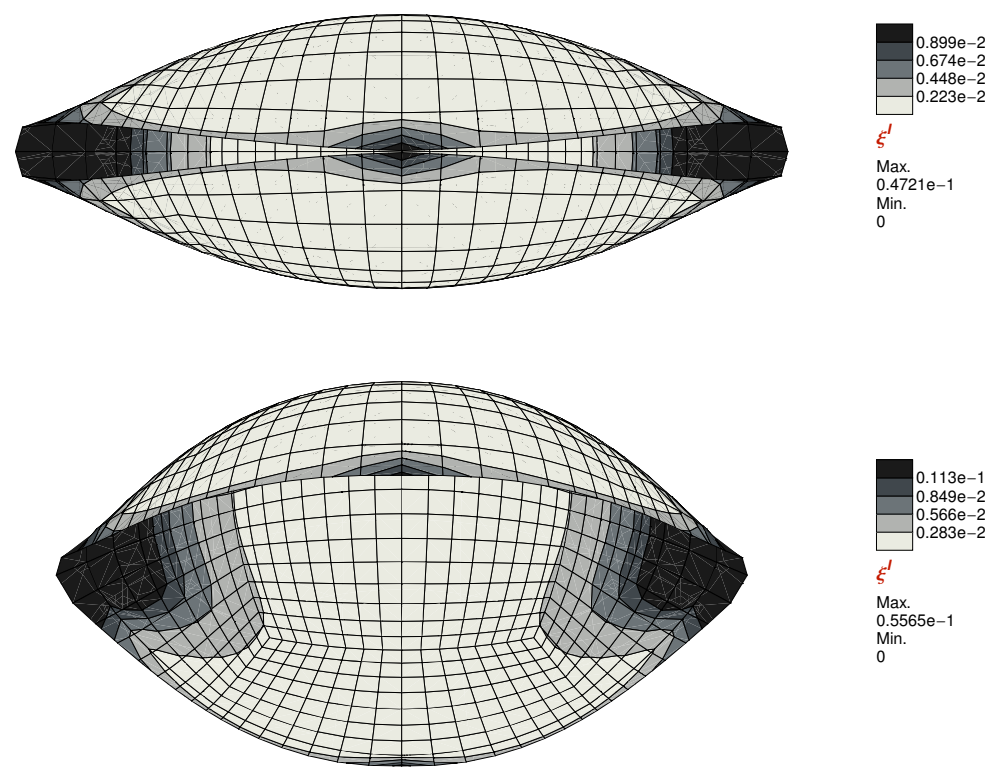

Figure 17: Deformed configuration with $\xi^{I}$ at $u_{A}=10 \mathrm{~cm}$ (top) and deformed configuration with $\xi^{I}$ after unloading (bottom) for $K_{h}=3 \mathrm{kN} / \mathrm{cm}^{2}$

\section{References}

[1] Hibbit, Karlsson, Sorensen, Abaqus manuals, Abaqus 6.7, 2007

[2] Başar Y, Itskov M, Constitutive model and finite element formulation for large strain elastoplastic analysis of shells, Computational Mechanics, 23, (1999), 466-481

[3] Bathe KJ, Dvorkin E (1985) A four-node plate bending element based on Mindlin-Reissner plate theory and a mixed interpolation, Int J Numer Meth Engng 21:367-383

[4] Brank B, Perić D, Damjanić FB (1997) On large deformation of thin elasto-plastic shells: implementation of a finite rotation model for quadrilateral shell elements, Int J Numer Meth Engng 40:689-726

[5] Brank B, Ibrahimbegovic A (2001) On the relation between different parametrizations of finite rotations for shells, Engineering Computations 18:950-973

[6] Dujc, J., Brank, B. 2008. On stress resultant plasticity and viscoplasticity for metal plates. Finite Elements in Analysis and Design, 44, 174-185, 2008.

[7] Ibrahimbegovic A, Brank B, Courtois P (2001) Stress resultant geometrically exact form of classical shell model and vector-like parametrization of constrained finite rotations, Int $\mathrm{J}$ Numer Meth Engng 52:1235-1252

[8] J. Korelc, Automation of primal and sensitivity analysis of transient coupled problems, Comput Mech 2009 44:631-649

[9] Korelc J (2012) AceGen, AceFem, available at http://www.fgg.uni-lj.si/Symech 


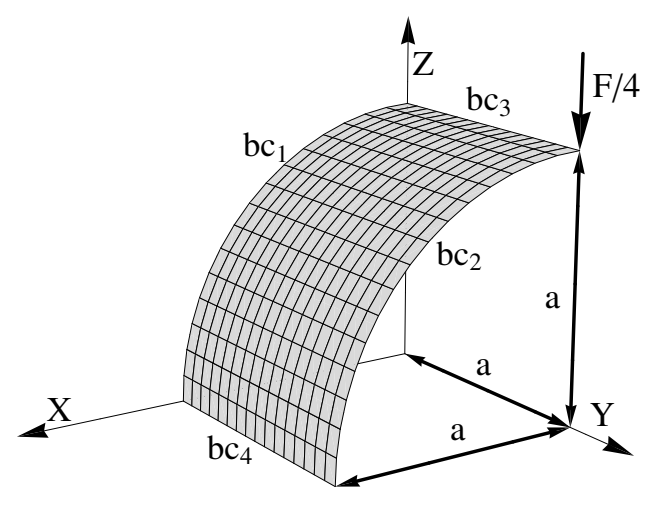

Figure 18: Geometry, loading and boundary conditions for pinched cylinder

[10] Simo JC, Kennedy JG. On a stress resultant geometrically exact shell model. Part V. Nonlinear plasticity: formulation and integration algorithms. Computer Methods in Applied Mechanics and Engineering 1992;96:133-171.

[11] Simo JC, Hughes TJR. Computational Inelasticity. Springer, 1998.

[12] Crisfield MA, Peng X. Efficient nonlinear shell formulations with large rotations and plasticity. In: D.R.J. Owen et al. Computational plasticity: models, software and applications, Part 1, Pineridge Press, Swansea, 1992. p. 1979-1997.

[13] Shi G, Voyiadjis GZ. A simple non-layered finite element for the elasto-plastic analysis of shear flexible plates. International Journal for Numerical Methods in Engineering 1992;33:85100.

[14] A. Ibrahimbegovic, Nonlinear Solid Mechanics: Theoretical Formulations and Finite Element Solution Methods, Springer, 2009.

[15] Voyiadjis, G.Z., Woelke, P. 2006. General non-linear finite element analysis of thick plates and shells. International Journal of Solidsand Structures, 43:2209-2242.

[16] Skallerud B, Myklebust LI, Haugen B. Nonlinear response of shell structures: effects of plasticity modelling and large rotations. Thin-Walled Structures 2001;39:463-482.

[17] P. Woelke, G. Z. Voyiadjis, P. Perzyna, Elasto-plastic finite element analysis of shells with damage due to microvoids, Int. J. Numer. Meth. Engng 2006; 68:338-380

[18] B. Skallerud, B. Haugen, Collapse of thin shell structures - stress resultant plasticity modelling with a co-rotated ADNES finite element formulation, Int. J. Numer. Meth. Engng. 46:1961-1986, 1999

[19] N. Dal Cortivo, C.A. Felippa, H. Bavestrello, W.T.M. Silva, Plastic buckling and collapse of thin shell structures, using layered plastic modeling and co-rotational ANDES finite elements, Computer Methods in Applied Mechanics and Engineering, 198: 785-798, 2009

[20] Q. Zeng, A. Combescure, F. Arnaudeau, An efficient plasticity algorithm for shell elements application to metal forming simulation, Computers and Structures, 79: 1525-1540, 2001 


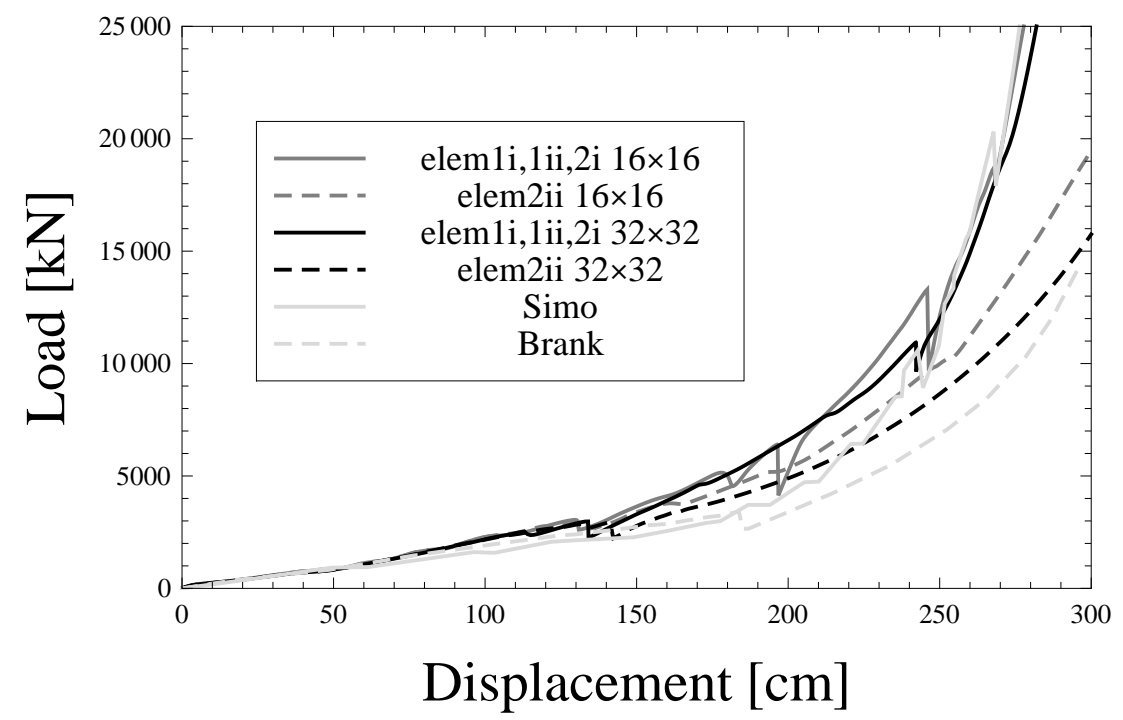

Figure 19: Load versus displacement curves for pinched cylinder

[21] W. Wagner W, F. Gruttmann, A robust non-linear mixed hybrid quadrilateral shell element, Int. J. Numer. Meth. Engng. 64:635-666, 2005

[22] K.D. Kim, G.R. Lomboy, A co-rotational quasi-conforming 4-node resultant shell element for large deformation elasto-plastic analysis, Comput. Methods Appl. Mech. Engrg. 195 (2006) 6502-6522

[23] J.C. Simo, D.D. Fox, M.S. Rifai, On a stress resultant geometrically exact shell model. Part III: Computational aspects of the nonlinear theory, Computer Methods in Applied Mechanics and Engineering, 79: 21-70, 1990

[24] P. L. Gould, Analysis of shells and plates, Springer, 1987

[25] P.M.A. Areias, J.-H. Song, T. Belytschko, A finite strain quadrilateral shell element based on discrete Kirchhoff-Love constraints, Int. J. Numer. Meth. Engng. 64:1166-1206, 2005

[26] B. Brank, Assessment of 4-node EAS-ANS shell elements for large deformation analysis, Comput. Mech. 42:39-51, 2008

[27] U. Bohinc, A. Ibrahimbegovic, B. Brank, Model adaptivity for finite element analysis of thin or thick plates based on equilibrated boundary stress resultants, Eng. Computations 26:66-99, 2009

[28] C. Kassiotis, A. Ibrahimbegovic, H.G. Matthies, B. Brank, Stable splitting svheme for general form of associated plasticity including different scales of space and time, Comput. Methods Appl. Mech. Engrg. 199:1254-1264, 2010

[29] A. Ghassemi, A. Shahidi, M. Farzin, A new element for analyzing large deformation of thin Naghdi shell model. Part II: Plastic, Applied mathematical modeling 35: 2650-2668, 2011 


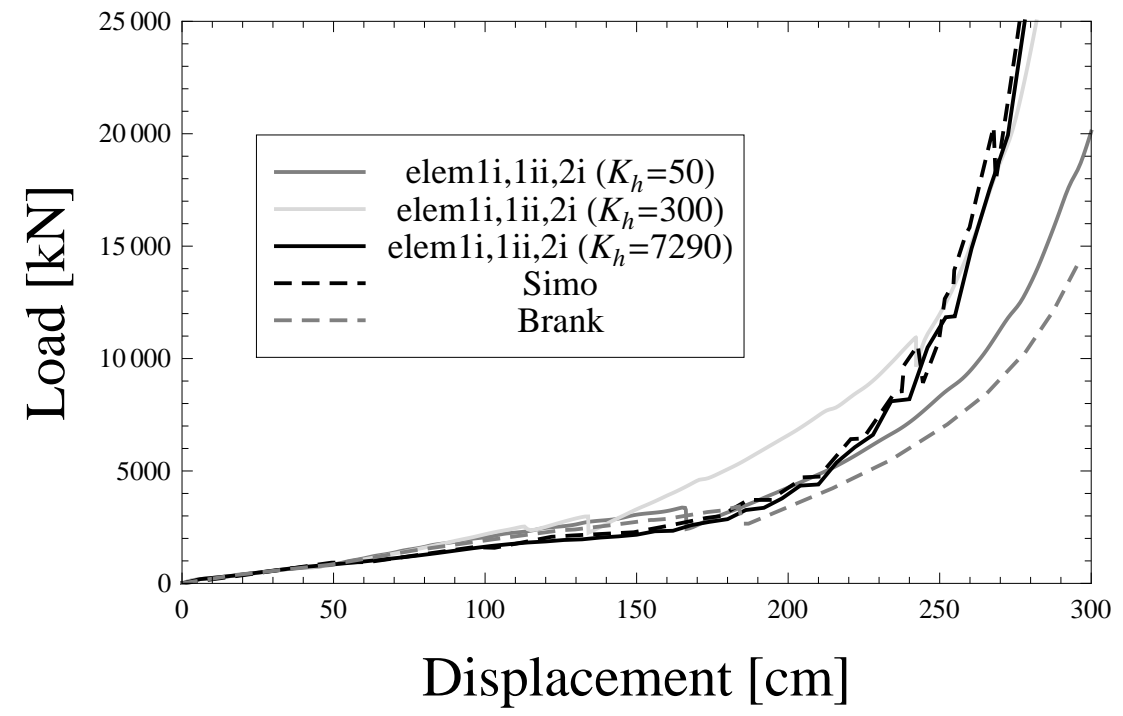

Figure 20: Load versus displacement curves for pinched cylinder $\left(K_{h}=0,50,100,300\right)$

[30] J.M.A. César de Sá, R.M. Natal Jorge. R.A. Fontes Valente, P.M. Almeida Areias, Development of shear locking-free shell elements using an enhanced assumed strain formulation, Int. J. Numer. Meth. Engng 53:1721-1750, 2002

[31] W. Wagner, S. Klinkel, F. Gruttmann, Elastic and plastic analysis of thin-walled structures using improved hexahedral elements, Comput. Struct. 80: 857-869, 2002

[32] F. Auricchio, R.L. Taylor, A generalized elastoplastic plate theory and its algorithmic implementation, Int. J. Numer. Meth. Engng, 37:2583-2608, 1994

[33] A. Ibrahimbegovic, F. Frey, Stress resultant finite element analysis of reinforced concrete plates, Eng. Computations 10:15-30, 1993

[34] A. Ibrahimbegovic, J.B. Colliat, L. Davenne, Thermomechanical coupling in folded plates and non-smooth shells, Comput. Methods Appl. Mech. Engrg. 194:2686-2707, 2005

[35] J.B. Colliat, A. Ibrahimbegovic, L. Davenne, Saint-Venant multi-surface plasticity model in strain space and stress resultants, Eng. Computations 22: 536-557, 2005

[36] K.-U. Bletzinger, M. Bischoff, E. Ramm, A unified approach for shear-locking-free triangular and rectangular shell finite elements, Comp. Struct. 75:321-334, 2000

[37] B. Brank, Nonlinear shell models with seven kinematic parameters, Comput. Methods Appl. Mech. Engrg. 194:2336-2363, 2005

[38] F. Cirak, M. Ortiz, Fully $\mathrm{C}^{1}$-conforming subdivision elements for finite deformation thinshell analysis, Int. J. Numer. Meth. Engng, 51:813-833, 2001

[39] P.M. Pimenta, E.M.B. Campello, Shell curvature as an initial deformation: A geometrically exact finite element approach, Int. J. Numer. Meth. Engng, 78:1094-1112, 2009 

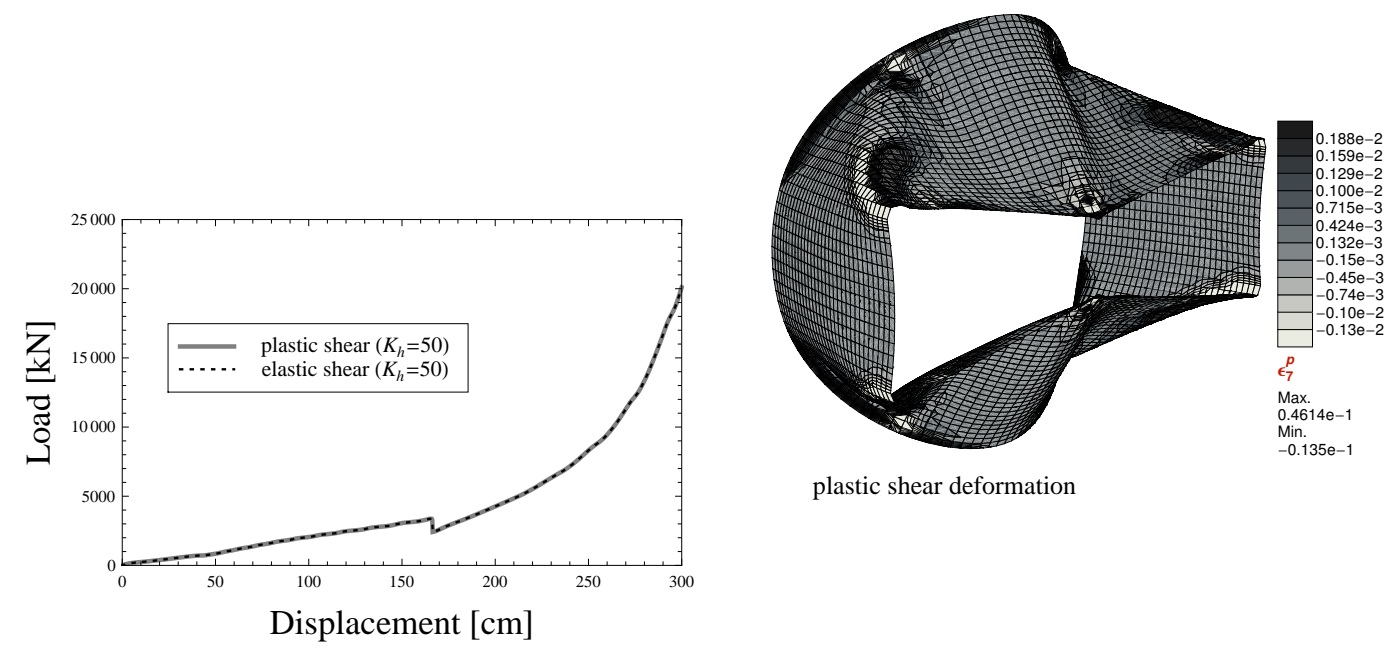

plastic shear deformation

Figure 21: Load versus displacement curves for pinched cylinder (left) and plastic shear strain $\widetilde{\gamma}_{1}^{p}$ (right)

[40] B. Brank, D. Perić, F.B. Damjanić, On implementation of a nonlinear four node shell finite element for multilayered elastic shells, Comput. Mechanics 16:341-359, 1995

[41] M. Bischoff, E. Ramm, Shear deformable shell elements for large strains and rotations, Int. J. Numer. Meth. Engng, 40:4427-4449, 1997

[42] P. Wriggers, R. Eberlein, F. Gruttmann, An axisymmetrical quasi-Kirchhoff-type shell element for large plastic deformations, Archive of Applied Mechanics 65:1-14, 1995

[43] P. Betsch, N. Sänger, On the use of geometrically exact shells in a conserving framework for flexible multibody dynamics, Comput. Methods Appl. Mech. Engrg. 198:1609-1630, 2009

[44] N. Büchter, E. Ramm, Shell theory versus degeneration - a comparison in large rotation shell analysis, Int. J. Numer. Methods Engrg. 34: 39-59, 1992.

[45] E. Ramm, W.A. Wall, Shell structures - a sensitive interrelation between physics and numerics, Int. J. Numer. Methods Engrg. 60: 381-427, 2004.

[46] K.Y. Sze, X.H. Liu, S.H. Lo, Popular benchmark problems for geometrically nonlinear analysis of shells, Finite Elements in Analysis and Design 40: 1551-1569, 2004.

[47] H.G. Matthies, A decomposition method for integration of the elastic-plastic rate problem, Int. J. Numer. Methods Engrg. 28: 1-11, 1989.

[48] P. Fuschi, D. Perić, D.R.J. Owen, Studies on generalized midpoint integration in rateindependent plasticity with reference to plane-stress $\mathrm{J}_{2}$-flow theory, Comput. Structures 43:1117-1133, 1992.

[49] J. Dujc, Finite element analysis of limit load and localized failure of structures, Doctoral Thesis, University of Ljubljana and ENS Cachan, 2010.

[50] M. A. Crisfield, X. Peng, Instabilities induced by coarse meshes for nonlinear shell problem, Engineering Computations 13:110-114, 1996. 


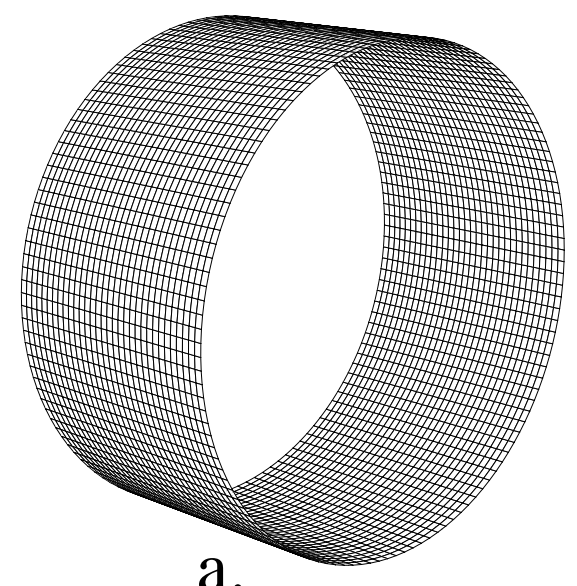

a.

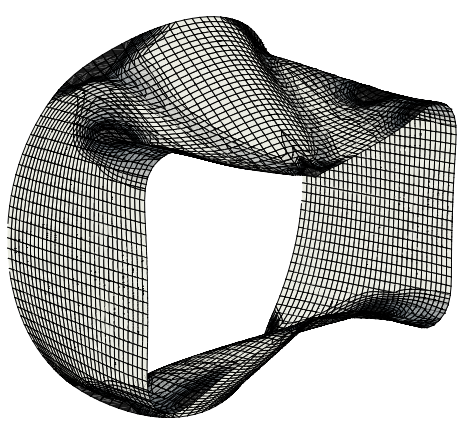

c.

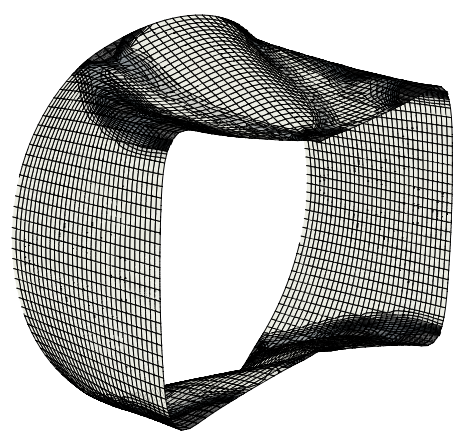

b.
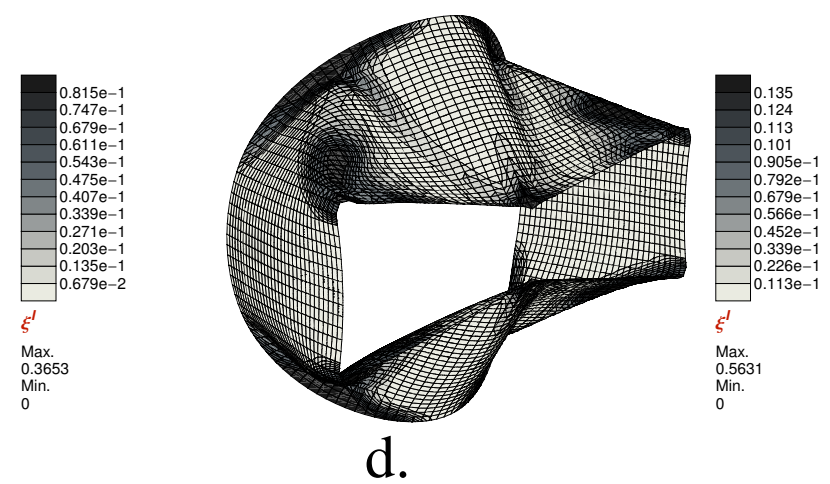

Figure 22: Initial configuration (a.), deformed configuration at $u_{Z}=150 \mathrm{~cm}$ (b.), deformed configuration at $u_{Z}=200 \mathrm{~cm}$ (c.) and deformed configuration at $u_{Z}=250 \mathrm{~cm}(\mathrm{~d}$.), 


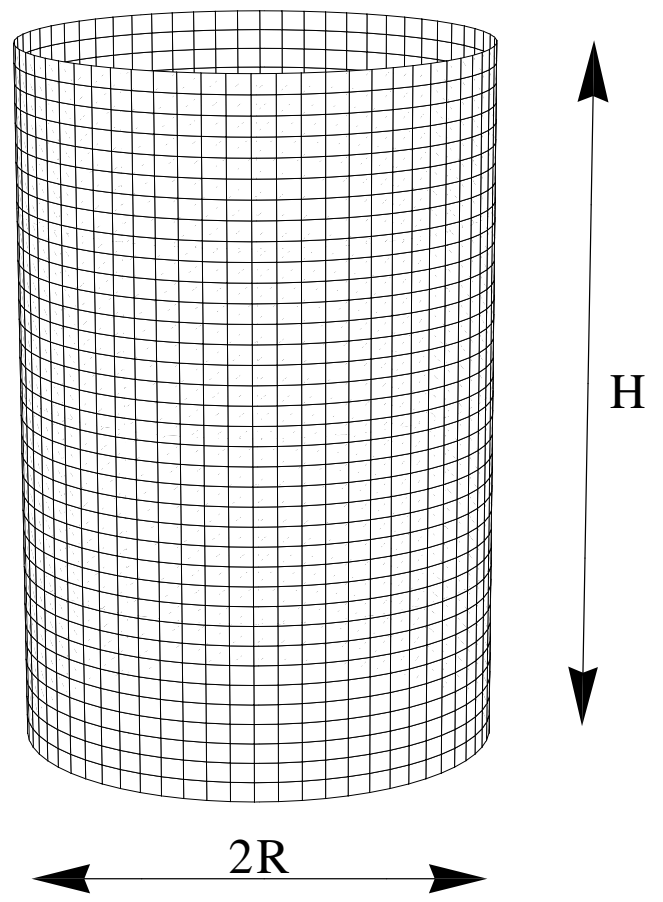

Figure 23: Finite element mesh of the cylinder

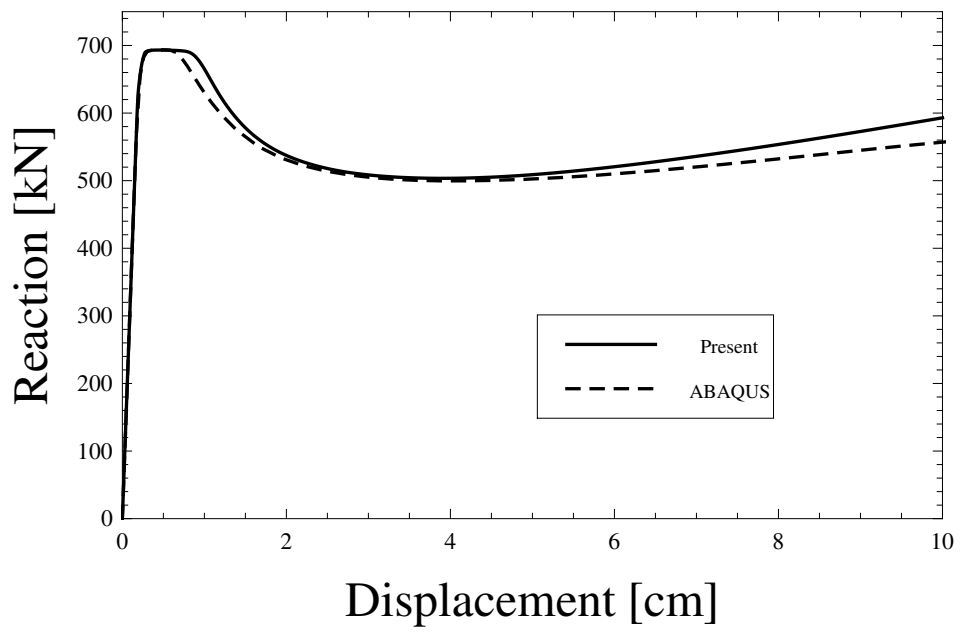

Figure 24: Total reaction ( $\mathrm{Y}$ direction) versus displacement curves 

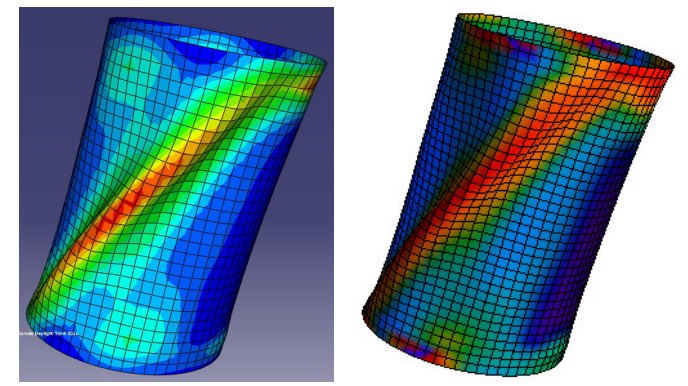

Figure 25: Deformed configurations at $u_{Y}=10 \mathrm{~cm}$ with equivalent plastic strain ABAQUS (left) and Present (right)

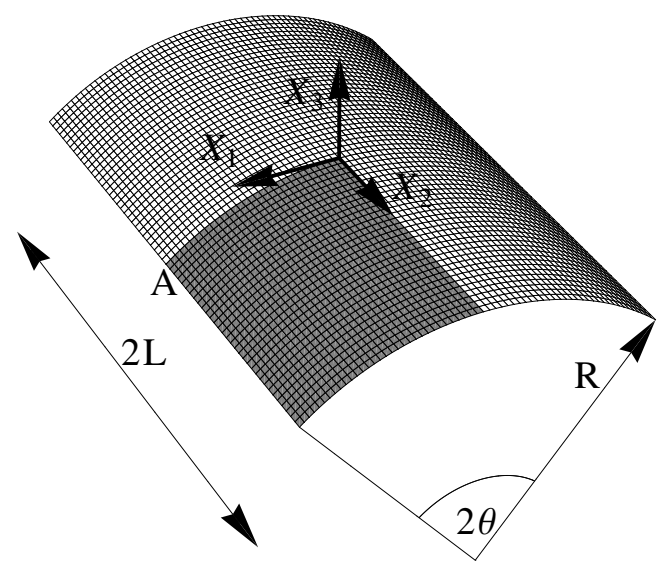

Figure 26: Scordelis-Lo roof geometry

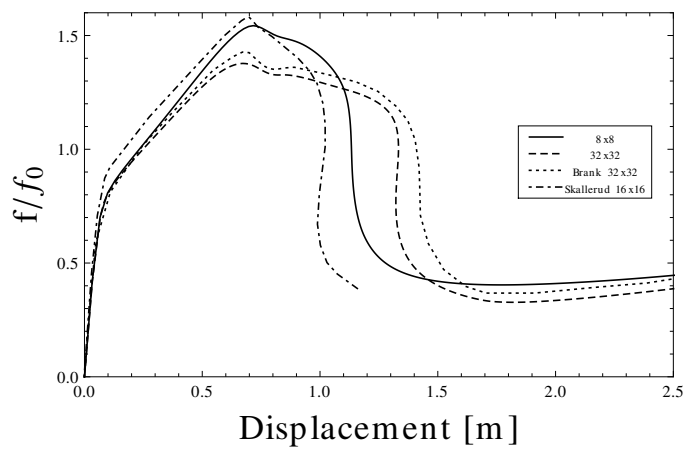

Figure 27: Load versus displacement curves for Scordelis-Lo roof 


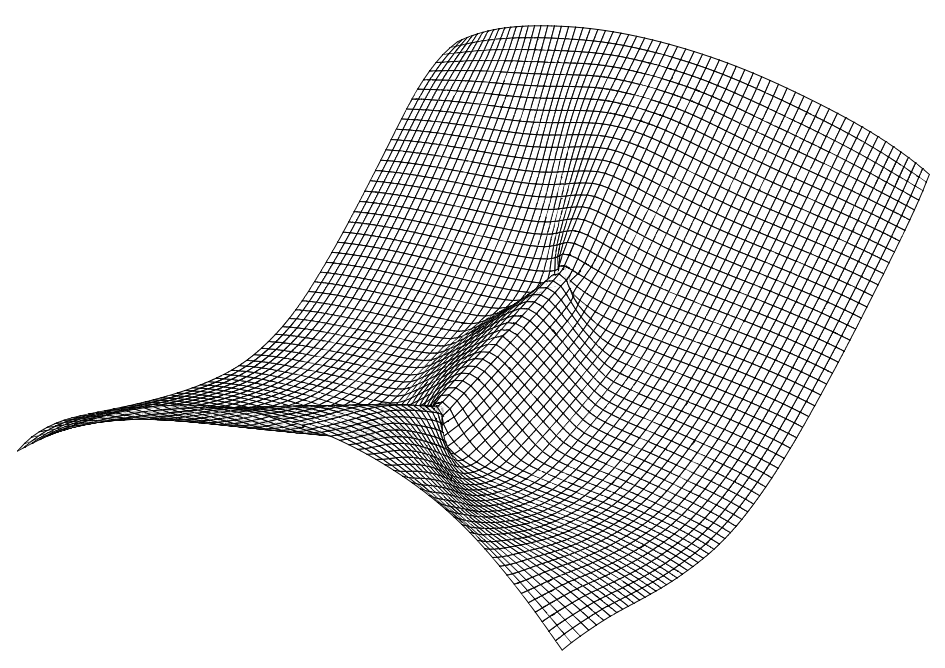

Figure 28: Deformed configuration for Scordelis-Lo roof at displacement equal to $2.5 \mathrm{~m}$ 